\title{
Does enrollment in multidisciplinary team-based primary care practice improve adherence to guideline-recommended processes of care? Quebec's Family Medicine Groups, 2002-2010
}

Diop, M. ${ }^{\mathrm{a}, \mathrm{b}}$,Fiset-Laniel, J. ${ }^{\mathrm{a}, \mathrm{b}}$, Provost, S. ${ }^{\mathrm{b}, \mathrm{c}}$, Tousignant, P. ${ }^{\mathrm{a}, \mathrm{b}}$, Borges da Silva, R. ${ }^{\mathrm{b}, \mathrm{d}}$, Ouimet, M.J. ${ }^{\mathrm{b}}$, Latimer, $E^{a}{ }^{a, e}$, Strumpf, E. ${ }^{a, b, c, f}$

${ }^{a}$ McGill University, Department of Epidemiology, Biostatistics and Occupational Health, 1020 Pine Ave. West, Montreal QC H3A 1A2 Canada

${ }^{b}$ Direction de santé publique du CIUSS du Centre-Sud-de-l'île-de-Montréal, 1301 Sherbrooke St. East, Montreal, QC H2L 1M3 Canada

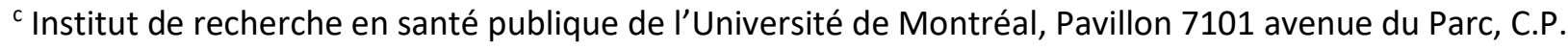
6128, Succ. Centre-Ville, Montreal QC H3C 3J7 Canada

${ }^{d}$ Université de Montréal, Faculté des sciences infirmières, Marguerite-d'Youville C.P 6128, succursale Centre-ville Montréal, QC H3C 3J7 Canada

e Douglas Mental Health University Institute, Perry Pavilion Room E-3114, 6875 boulevard LaSalle, Montreal, QC H4H 1R3 Canada

${ }^{f}$ McGill University, Department of Economics, 855 Sherbrooke St. West, Leacock 418, Montreal, QC H3A 2T7 Canada

\section{Abstract}

Background: We investigated whether multidisciplinary team-based primary care practice improves adherence to process of care guidelines, in the absence of financial incentives related to pay-forperformance.

Methods: We conducted a natural experiment including 135,119 patients, enrolled with a general practitioner (GP) in a multidisciplinary team Family Medicine Group (FMG) or non-FMG practice, using longitudinal data from Quebec's universal insurer over the relevant time period (2000-2010). All study subjects had diabetes, chronic obstructive pulmonary disease, or heart failure and were followed over a 7-year period, 2 years prior to enrollment and 5 years after. We constructed indicators on adherence to disease-specific guidelines and composite indicators across conditions. We evaluated the effect of FMGs using propensity score methods and Difference-in-Differences (DD) models.

Does enrollment in multidisciplinary team-based primary care practice improve adherence to guidelinerecommended processes of care? Quebec's Family Medicine Groups, 2002-2010 M Diop, J Fiset-Laniel, S Provost, P Tousignant, RB Da Silva, MJ Ouimet. Health Policy 121 (4), 378-388 
Results: Rates of adherence to chronic disease guidelines increased for both FMG and non-FMG patients after enrollment, but not differentially so. Adherence to prescription-related guidelines improved less for FMG patients (DD $[95 \% \mathrm{Cl}]=-2.83 \%[-4.08 \%,-1.58 \%])$. We found no evidence of an FMG effect on adherence to consultation-related guidelines, (DD [95\% Cl] = - $0.24 \%[-2.24 \% ; 1.75 \%])$.

Conclusions: We found no evidence that FMGs increased adherence to the guidelines we evaluated. Future research is needed to assess why this reform did not improve performance on these quality-ofcare indicators.

Keywords: clinical guideline adherence; team-based primary care; quality of care; chronic disease; difference-in-differences

Highlights for Health Policy:

- Adherence to clinical guidelines for 3 chronic diseases increased after enrollment with any GP

- Enrollment in team-based practices decreased adherence to prescription drug-related guidelines

- No evidence that team-based practices affect adherence to consultation-related guidelines

\section{CONTEXT}

The burden of chronic disease and process-of-care guidelines

Chronic diseases are the leading cause of mortality and morbidity around the world(1, 2). As elsewhere, chronic disease prevalence follows an upward trend in Canada and Quebec, the second most populous Canadian province (23\% of the Canadian population)(3). In 2009-2010, 44\% of Quebecers aged 20 and over had at least one chronic disease(4).

Chronic diseases and their complications are associated with an increased risk of death and a deterioration in quality of life(5-8). Several studies have shown the health benefits associated with proper management of chronic diseases(9-12) and the relationship between better processes of ambulatory care and better health. These findings underscore the importance of efforts to improve quality of care, which for several chronic diseases means processes of care that are consistent with clinical guidelines(10). We

Does enrollment in multidisciplinary team-based primary care practice improve adherence to guidelinerecommended processes of care? Quebec's Family Medicine Groups, 2002-2010 M Diop, J Fiset-Laniel, S Provost, P Tousignant, RB Da Silva, MJ Ouimet. Health Policy 121 (4), 378-388 
focus on adherence to current Canadian guidelines for diabetes, heart failure (HF) and chronic obstructive pulmonary disease (COPD) (Table 1).

Table 1: Clinical guidelines

\begin{tabular}{|c|c|c|c|c|}
\hline Disease & Guideline & $\begin{array}{l}\text { Target } \\
\text { Age }\end{array}$ & Objective & Source \\
\hline \multirow{3}{*}{ Diabetes } & Prescribe statins & $\begin{array}{l}40 \text { and } \\
\text { over }\end{array}$ & $\begin{array}{l}\text { Reduce the risk } \\
\text { of } \\
\text { cardiovascular } \\
\text { events }\end{array}$ & $\begin{array}{l}\text { Canadian Diabetes } \\
\text { Association }(8,13,14)\end{array}$ \\
\hline & $\begin{array}{l}\text { Prescribe angiotensin- } \\
\text { converting enzyme inhibitors } \\
\text { (ACEI) or angiotensin receptor } \\
\text { blockers (ARBs) }\end{array}$ & $\begin{array}{l}\text { Men: } 45 \\
\text { and older } \\
\text { Women: } \\
50 \text { and } \\
\text { older }\end{array}$ & $\begin{array}{l}\text { Reduce the risk } \\
\text { of } \\
\text { cardiovascular } \\
\text { events }\end{array}$ & $\begin{array}{l}\text { Canadian Diabetes } \\
\text { Association }(8,13,14)\end{array}$ \\
\hline & $\begin{array}{l}\text { Annual or biannual screening } \\
\text { for retinopathy }\end{array}$ & NA & $\begin{array}{l}\text { Early diagnosis } \\
\text { and treatment } \\
\text { of retinopathy }\end{array}$ & $\begin{array}{l}\text { Canadian Diabetes } \\
\text { Association }(8,13,14)\end{array}$ \\
\hline \multirow{2}{*}{$\begin{array}{l}\text { Heart } \\
\text { Failure }\end{array}$} & $\begin{array}{l}\text { Prescribe } A C E I \text { in combination } \\
\text { with a beta-blocker or ARB }\end{array}$ & NA & $\begin{array}{l}\text { Pro-actively } \\
\text { manage } \\
\text { cardiovascular } \\
\text { risks }\end{array}$ & $\begin{array}{l}\text { Canadian Cardiovascular } \\
\text { Society }(5,6)\end{array}$ \\
\hline & $\begin{array}{l}\text { Regular visits to a general } \\
\text { practitioner or a cardiologist }\end{array}$ & NA & $\begin{array}{l}\text { Proper disease } \\
\text { management }\end{array}$ & $\begin{array}{l}\text { Canadian Cardiovascular } \\
\text { Society (5) }\end{array}$ \\
\hline COPD & $\begin{array}{l}\text { Do not use inhaled } \\
\text { corticosteroids as } \\
\text { monotherapy }\end{array}$ & NA & $\begin{array}{l}\text { Reduce } \\
\text { symptoms; } \\
\text { improve activity } \\
\text { levels and } \\
\text { quality of life }\end{array}$ & $\begin{array}{l}\text { Canadian Thoracic } \\
\text { Society (15-17) }\end{array}$ \\
\hline
\end{tabular}

Research on models of primary care organization, financial incentives, and adherence to clinical guidelines

Several studies have examined the possible influence of organizational characteristics and financial incentives in primary care on quality of care, particularly adherence to guidelines. A cross-sectional study in Ontario(18) indicates that chronic disease management is better in multidisciplinary primary care models because of inter-professional collaboration and longer consultations and that the presence of a Does enrollment in multidisciplinary team-based primary care practice improve adherence to guidelinerecommended processes of care? Quebec's Family Medicine Groups, 2002-2010 M Diop, J Fiset-Laniel, S Provost, P Tousignant, RB Da Silva, MJ Ouimet. Health Policy 121 (4), 378-388 
nurse is associated with higher levels of chronic disease management, independent of the organizational model. A Quebec study found that integrated and community models are more likely to provide care consistent with quality process measures(19). In Alberta, diabetic patients followed in primary care networks receive better care and show improved clinical outcomes compared to diabetics who are not followed in a network; however, the differences are very small(20). Other studies in United Kingdom and United States found a positive association between quality of care and multidisciplinary group primary care organizations(21-25) However, except for Manns et al.(20), they all report associations not causal relationships.

Beyond the organizational structure they practice in, general practitioners' (GPs) attitudes towards evidence-based guidelines and their compensation mechanisms are predictive of their practice behavior(26). For these reasons, public and private decision-makers also adopt payment mechanisms based on physician performance to improve the quality of care(27). Some evidence supports the idea that such incentives can have a positive impact on quality of care(28-31). However, most studies we reviewed provide mixed results or weaker evidence(32-40). Town et al.(40) and Allen et al.(41) conclude that there is little evidence that the small financial rewards offered to date impact physicians' preventive care routines, but that more research is needed to understand how effective other types or amounts of incentives might be. It is also important to note that because financial incentives are often bundled together with the introduction of group-based, multidisciplinary practice, it is difficult to understand what the latter may contribute separately to improving adherence to clinical guidelines.

\section{The Family Medicine Group reform in Quebec}

The creation of multidisciplinary team-based primary care practices in Quebec, known as Family Medicine Groups (FMGs), began in November 2002. The main objectives of the FMG policy were to 
improve accessibility and quality of care, one important component of which is adherence to guidelines for chronic disease management. FMGs are intended to serve as a medical home for enrolled patients and to provide case management, follow-up, and health promotion services for patients in their geographical area $(19,42,43)$. Based on the evidence from multidisciplinary team-based practices like that reviewed above, FMGs are expected to improve quality of care, especially for patients with chronic conditions. Both physician participation and patient enrollment in FMGs are voluntary.

FMGs were designed to include 6-12 full-time equivalent GPs who enroll 1,000-2,200 patients each and work in collaboration with nurses(43-46). FMG practices receive additional funding for nurses, administrative support staff, and computer equipment and agree to increase after-hours access. In their qualitative examination of the implementation of 5 early FMGs, Beaulieu et al. cite the importance of physician leadership, collaborative care protocols, and formal meetings in developing effective group practice, while also acknowledging the wide variation across FMGs in their structure and functioning(42). FMG GPs can bill a small annual fee for the management of enrolled patients. Otherwise they have the same incentives as non-FMG GPs to enroll vulnerable (elderly or chronically ill, Appendix 1)(47) patients and they have the same mode of remuneration, largely fee-for-service. FMGs generally resemble teambased primary care models in other jurisdictions: Family Health Teams and Family Health Groups in Ontario, Patient-Centered Medical Homes in the United States, Multidisciplinary Medical Homes in France, and General Practitioner Fund Holders in the United Kingdom. However, FMGs do not share certain characteristics that are often found in these other models, including pay-for-performance incentives, mixed remuneration, a diverse range of non-physician health professionals, or full implementation of electronic medical records(48).

As of March 31, 2014 there were 258 FMGs in Quebec, including nearly 4,200 participating GPs (55\%), nearly 600 nurses, and more than three million people (42\% of Quebec's population)(49). Not all policy

Does enrollment in multidisciplinary team-based primary care practice improve adherence to guidelinerecommended processes of care? Quebec's Family Medicine Groups, 2002-2010 M Diop, J Fiset-Laniel, S Provost, P Tousignant, RB Da Silva, MJ Ouimet. Health Policy 121 (4), 378-388 
goals have been achieved, however. In its report, the Auditor General of Quebec(50) indicated that at the end of 2014, 43\% of FMGs had not enrolled the required number of patients, and that computerization was behind schedule, with $73 \%$ of FMG practices relying on electronic medical records.

\section{New Contribution}

We evaluate the effect of multidisciplinary primary care teams on adherence to clinical guidelines. More specifically, we examine if enrollment with a GP in an FMG increases the probability that patients' care is consistent with clinical guidelines for three chronic diseases, compared to patients enrolled with GPs in non-team-based practices. A large number of innovative initiatives have been introduced to improve clinical practice prevention and chronic disease management. Relatively few have been evaluated in ways that allow an understanding of their causal effects on quality of care, particularly among population-level policy interventions(4). We address these limitations with a quasi-experimental design that allows us to estimate the causal effects of the policy change. The time period covered by our analysis includes a follow-up period that is reasonably adequate to detect any impacts on guideline adherence, while not extending so far from the policy change that we capture spurious differences between the treatment and control groups. Furthermore, most studies of the effects of multidisciplinary models on guideline-recommended care are implemented in contexts where reforms include financial incentives. Our study analyzes the effects of organizational reforms in the absence of pay-for-performance-style financial incentives and mixed remuneration, permitting a better understanding of their impact in isolation and providing useful information to decision makers considering bundled or unbundled primary care reforms.

Does enrollment in multidisciplinary team-based primary care practice improve adherence to guidelinerecommended processes of care? Quebec's Family Medicine Groups, 2002-2010 M Diop, J Fiset-Laniel, S Provost, P Tousignant, RB Da Silva, MJ Ouimet. Health Policy 121 (4), 378-388 


\section{METHODS}

\section{Design, data, and study population}

We conducted a cohort study including individuals with at least one chronic disease for which adherence to guidelines was measurable in our administrative database (diabetes, COPD or HF). We compared changes in guideline-consistent care in the treatment group - patients enrolled in multidisciplinary teams (FMGs) - to changes in the control group - patients enrolled in non-team-based primary care. Patient characteristics between the two groups were made comparable using propensity score (PS) adjustment.

Our analyses used population-based, insurance billing data from Quebec's provincial public insurer, the Régie de l'assurance maladie du Québec (RAMQ). The RAMQ insures all physician and hospital services for about $96 \%$ of the Quebec population(51) and prescription drugs for about $90 \%$ of residents aged 65 and over, with the remainder covered by private insurance or Federal programs(52). These data contain information on individual-level demographics and health care services utilization including physicians' services, hospital admissions, emergency department visits, same-day surgeries and prescriptions filled. Our database includes all elderly (age $70+$ ) or chronically ill patients who enrolled with a primary care physician from November 2002 to January 2005. Our analysis includes the first 79 FMGs created in Quebec, and understanding their experiences vis-à-vis quality of care is important to inform the iterative process of primary care reform that is ongoing there and in other jurisdictions. Because of financial incentives for all physicians to enroll "vulnerable" patients in their practice, we expect that our database includes the near-population of older and chronically ill patients with at least one physician visit over this period. Our database comprises 7 years of data for each individual: 2 years prior to and 5 years after their enrollment with a primary care physician.

Does enrollment in multidisciplinary team-based primary care practice improve adherence to guidelinerecommended processes of care? Quebec's Family Medicine Groups, 2002-2010 M Diop, J Fiset-Laniel, S Provost, P Tousignant, RB Da Silva, MJ Ouimet. Health Policy 121 (4), 378-388 
Our database includes a closed cohort of 797,248 vulnerable patients (Appendix 2). We exclude patients who live in remote northern areas of Quebec (Nord-du-Québec, Nunavik and Cree Territories of James Bay). Additionally, those who died or moved to another type of geographic area during follow-up, resided in a long-term care facility, and those with missing or invalid data are also excluded. We identified patients with our three target chronic conditions using algorithms developed and validated by the Quebec National Institute of Public Health (Appendix 3)(53,54). We restricted our sample to 135,119 patients who had at least one of the target diseases and met the guidelines' age criteria at the time of enrollment with a GP. The final analytic samples were 35,088 diabetics aged 65 and over, 6,832 patients with COPD aged 65 and over and taking steroids at baseline, 4,928 HF patients aged 65 and older, and 5,952 HF patients aged 40 and over (Appendix 2).

\section{Variables}

\section{Dependent variables}

We created two categories of guideline adherence indicators: 1) specific indicators relating to prescription drugs or consultations by chronic disease and 2) composite indicators, combining the specific indicators applicable to the same individual across multiple chronic conditions (Appendix 4). All adherence indicators are calculated over two-year periods. They are based on specific age groups as per the clinical guidelines and those for prescription drugs are based on patients age 65 and over.

\section{Exposure variables}

We created two indicator variables: one denoting being in the treatment group (enrollment with a FMG GP at the time of enrollment, $F M G=1)$ or not $(F M G=0)$, another indicating the period after enrollment $($ Post $=1)$ or before $($ Post $=0)$. The exposure is being in the treatment group in the post-enrollment period, represented by the interaction of FMG and Post.

Does enrollment in multidisciplinary team-based primary care practice improve adherence to guidelinerecommended processes of care? Quebec's Family Medicine Groups, 2002-2010 M Diop, J Fiset-Laniel, S Provost, P Tousignant, RB Da Silva, MJ Ouimet. Health Policy 121 (4), 378-388 


\section{Control variables}

The PS and DD models include several demographic characteristics: sex, 5-year age groups, geographic region, socioeconomic status, RAMQ coverage for prescription drugs, and calendar year. Region corresponds to Quebec's health regions grouped into four categories(55) and socioeconomic status is measured according to the quintile of the Pampalon index of economic deprivation(56). RAMQ coverage for prescription drugs is determined according to an algorithm based on age, prescription drug use, and the patient's level of morbidity (the Johns Hopkins ACG Case-Mix System Resource Utilization Bands (RUB)). Health care utilization variables include whether the patient has a usual provider of care (UPC) and the numbers of: outpatient physician visits, emergency department (ED) visits for all causes, ED visits and hospital admissions for four ambulatory care sensitive conditions, unique physicians consulted, prescriptions, and days taking prescribed medicines. Health status is captured using indicators for nine different chronic conditions and the 6-category RUB. See Appendix 1 for details.

\section{Analyses}

To strengthen our quasi-experimental difference-in-differences (DD) design, we use a PS approach to make the treated and control groups as similar as possible before exposure, thereby reducing the potential for bias due to confounding given voluntary participation in the new FMG model(57-59). We estimate a logistic regression of FMG enrollment on the following potential confounders measured in the year before enrollment: sex, age, geographic region, socioeconomic status, RUB, chronic conditions, health care utilization, having a UPC, RAMQ coverage for prescription drugs, and indicators for whether care was consistent with relevant clinical guidelines for patients' index conditions. The resulting predicted probability that each individual would enroll in a FMG is their PS. The sample was restricted to the common support of the PS distributions for the two groups and then weighted with inverse probability of

Does enrollment in multidisciplinary team-based primary care practice improve adherence to guidelinerecommended processes of care? Quebec's Family Medicine Groups, 2002-2010 M Diop, J Fiset-Laniel, S Provost, P Tousignant, RB Da Silva, MJ Ouimet. Health Policy 121 (4), 378-388 
treatment weights (IPTW)(57, 60). We preferred IPTW to the often-used matching methods since it can render the treatment and control groups comparable without sacrificing sample size, allowing for greater generalizability and policy relevance.

To evaluate the effect of enrollment of elderly or chronically ill patients in FMGs on guideline adherence, we estimated the following DD model:

$Y_{i j t}=\beta_{0}+\beta_{1} F M G_{j}+\beta_{2}$ Post $_{t}+\beta_{3} F M G_{j} \times$ Post $_{t}+\beta_{4} X_{i j t}+\varepsilon_{i j t}$

$Y_{i j t}$ is an indicator for adherence to guidelines for patient $i$ in group $j$ at time $t . F M G_{j}$ is an indicator for being enrolled with a FMG physician and therefore $\beta_{1}$ captures any time-invariant differences that remain between the treated and control groups after IPTW adjustment. Changes over time between the pre- and post-enrollment periods that are common to both groups are captured in $\beta_{2}$. Our coefficient of interest is $\beta_{3}$, which captures any additional change over time for the FMG patients relative to the change over time for the non-FMG patients. Individual-level control variables are included in the vector $X_{i j t}$ : sex, age, socioeconomic status, geographic region, and a set of indicators for calendar year, which control flexibly for any time trends common to both groups. RAMQ coverage for prescription drugs is also included as a control variable in models examining adherence to prescription drug-related guidelines.

Using the DD model to estimate causal effects requires several important assumptions. Most importantly, the control group serves as a counterfactual for what would have happened to the treatment group in the absence of treatment, so the two groups must be exchangeable. In order to interpret $\beta_{3}$ as the effect of FMG enrollment, there must be no other factor that changes at the same time as enrollment with a GP that affects only one group $(61,62)$. We are not able to assess the similarity of pre-intervention

Does enrollment in multidisciplinary team-based primary care practice improve adherence to guidelinerecommended processes of care? Quebec's Family Medicine Groups, 2002-2010 M Diop, J Fiset-Laniel, S Provost, P Tousignant, RB Da Silva, MJ Ouimet. Health Policy 121 (4), 378-388 
outcome trends, so we use IPTW to make these assumptions more reasonable and address both of them empirically in the results section.

We use multivariate logistic regressions and GEE since the number of observation periods is small(63) and we observed strong intra-individual autocorrelation in the dependent variables. For ease of interpretation, we transformed estimated odds ratios to marginal effects on the absolute scale. All analyses were conducted using Stata 13 software.

\section{RESULTS}

\section{Patient characteristics}

Unadjusted baseline patient characteristics are somewhat different between FMG and non-FMG patients (Table 2). FMG patients are more likely to live in peripheral and intermediate regions than nonFMG patients. This reflects regional variation in the rate of development of FMGs(64). A higher proportion of more socioeconomically advantaged patients and those with a UPC enrolled in non-FMG clinics. We estimate that almost all patients aged 65 or older have RAMQ drug coverage, equal in both groups. While many characteristics were statistically significantly different between the two groups in the unweighted sample, no significant or substantive differences remain after PS adjustment using IPTW (Table 2). This strong similarity of the two groups before exposure based on observed characteristics lends strength to our exchangeability assumption. Baseline characteristics for additional patient subsamples are in Appendix 5.

Does enrollment in multidisciplinary team-based primary care practice improve adherence to guidelinerecommended processes of care? Quebec's Family Medicine Groups, 2002-2010 M Diop, J Fiset-Laniel, S Provost, P Tousignant, RB Da Silva, MJ Ouimet. Health Policy 121 (4), 378-388 
Table 2: Baseline patient characteristics of patients in analysis sample

\begin{tabular}{|c|c|c|c|c|c|c|c|c|c|c|c|c|}
\hline & \multicolumn{4}{|c|}{ Diabetes $65+$} & \multicolumn{4}{|c|}{ Heart failure $65+$} & \multicolumn{4}{|c|}{ COPD de $65+$} \\
\hline & \multicolumn{2}{|c|}{ Unweighted } & \multicolumn{2}{|c|}{ Weighted } & \multicolumn{2}{|c|}{ Unweighted } & \multicolumn{2}{|c|}{ Weiahted } & \multicolumn{2}{|c|}{ Unweighted } & \multicolumn{2}{|c|}{ Weighted } \\
\hline & FMG & non-FMG & FMG & non-FMG & FMG & non-FMG & FMG & non-FMG & FMG & non-FMG & FMG & non-FMG \\
\hline & $(n=5,069)$ & $(n=30,019)$ & $(n=5,069)$ & $(n=30,019)$ & $(n=764)$ & $(n=4,164)$ & $(n=764)$ & $(n=4,164)$ & $(n=896)$ & $(n=5,936)$ & $(n=896)$ & $(n=5,936)$ \\
\hline & $\%(S E)$ & $\%(S E)$ & $\%(S E)$ & $\%(S E)$ & $\%(S E)$ & $\%(S E)$ & $\%(S E)$ & $\%(S E)$ & $\%(S E)$ & $\%(S E)$ & $\%(S E)$ & $\%(S E)$ \\
\hline \multicolumn{13}{|l|}{ Sex } \\
\hline Male & $45.8(0.7)$ & $46.7(0.3)$ & $45.2(0.8)$ & $46.8(0.3)$ & $45.4(1.8)$ & $45.9(0.8)$ & $44.6(2.0)$ & $46.3(0.8)$ & $48.7(1.7)$ & $50.7(0.7)$ & $46.9(1.8)$ & $50.9(0.7)$ \\
\hline \multicolumn{13}{|l|}{ Age categories } \\
\hline 60 to 74 years & $64.4(0.7)$ & $63.5(0.3)$ & $63.3(0.8)$ & $63.6(0.3)$ & $46.3(1.8)$ & $43.8(0.8)$ & $45.6(2.0)$ & $44.1(0.8)$ & $54.5(1.7)$ & $55.1(0.7)$ & $53.7(1.8)$ & $55.2(0.7)$ \\
\hline$>=75$ years & $35.6(0.7)$ & $36.5(0.3)$ & $36.7(0.8)$ & $36.4(0.3)$ & $53.7(1.8)$ & $56.2(0.8)$ & $54.4(2.0)$ & $55.9(0.8)$ & $45.5(1.7)$ & $44.9(0.7)$ & $46.3(1.8)$ & $44.8(0.7)$ \\
\hline \multicolumn{13}{|l|}{ Geographic Regions } \\
\hline University Region & $23.1(0.6)$ & $42.0(0.3)$ & $36.5(0.7)$ & $38.5(0.2)$ & $23.3(1.5)$ & $37.8(0.8)$ & $36.5(2.1)$ & $35.4(0.7)$ & $22.0(1.4)$ & $34.1(0.6)$ & $33.6(1.9)$ & $32.3(0.6)$ \\
\hline Peripheral Region & $45.8(0.7)$ & $36.0(0.3)$ & $40.0(0.6)$ & $37.4(0.2)$ & $46.2(1.8)$ & $37.5(0.8)$ & $40.2(1.9)$ & $38.5(0.8)$ & $36.9(1.6)$ & $37.3(0.6)$ & $35.8(1.7)$ & $37.5(0.6)$ \\
\hline Intermediate Region & $25.8(0.6)$ & $18.2(0.2)$ & $19.5(0.4)$ & $19.8(0.2)$ & $24.6(1.6)$ & $19.0(0.6)$ & $18.2(1.3)$ & $20.2(0.6)$ & $33.4(1.6)$ & $23.0(0.6)$ & $24.3(1.4)$ & $24.4(0.6)$ \\
\hline Remote Region & $5.4(0.3)$ & $3.8(0.1)$ & $4.1(0.2)$ & $4.3(0.1)$ & $5.9(0.9)$ & $5.7(0.4)$ & $5.1(0.8)$ & $5.9(0.4)$ & $7.7(0.9)$ & $5.6(0.3)$ & $6.3(0.8)$ & $5.8(0.3)$ \\
\hline \multicolumn{13}{|c|}{ Pampalon's Material Deprivation Index } \\
\hline 1 (advantaged) & $11.4(0.5)$ & $15.1(0.2)$ & $15.0(0.6)$ & $14.5(0.2)$ & $10.3(1.1)$ & $15.4(0.6)$ & $13.7(1.5)$ & $14.7(0.5)$ & $8.7(0.9)$ & $12.0(0.4)$ & $11.1(1.2)$ & $11.5(0.4)$ \\
\hline 2 & $19.4(0.6)$ & $18.2(0.2)$ & $18.9(0.6)$ & $18.2(0.2)$ & $17.4(1.4)$ & $18.1(0.6)$ & $18.3(1.5)$ & $17.9(0.6)$ & $15.7(1.2)$ & $15.6(0.5)$ & $16.0(1.3)$ & $15.6(0.5)$ \\
\hline 3 & $22.2(0.6)$ & $21.1(0.2)$ & $21.0(0.6)$ & $21.3(0.2)$ & $21.5(1.5)$ & $19.3(0.6)$ & $19.2(1.5)$ & $19.7(0.6)$ & $20.8(1.4)$ & $21.3(0.5)$ & $19.1(1.4)$ & $21.7(0.5)$ \\
\hline 4 & $25.2(0.6)$ & $22.7(0.2)$ & $23.4(0.7)$ & $23.1(0.3)$ & $23.0(1.5)$ & $23.0(0.7)$ & $20.8(1.6)$ & $23.4(0.7)$ & $27.5(1.5)$ & $22.9(0.6)$ & $25.8(1.6)$ & $23.2(0.6)$ \\
\hline 5 (disadvantaged) & $21.8(0.6)$ & $22.9(0.2)$ & $21.8(0.6)$ & $23.0(0.2)$ & $27.8(1.6)$ & $24.3(0.7)$ & $28.1(1.8)$ & $24.3(0.7)$ & $27.3(1.5)$ & $28.1(0.6)$ & $28.1(1.7)$ & $27.9(0.6)$ \\
\hline \multicolumn{13}{|l|}{ Health status } \\
\hline Diabetes & 100 & 100 & 100 & 100 & $28.4(1.6)$ & $27.3(0.7)$ & $26.9(1.7)$ & $27.7(0.7)$ & $15.1(1.2)$ & $15.2(0.5)$ & $14.2(1.2)$ & $15.3(0.5)$ \\
\hline COPD & $9.6(0.4)$ & $9.3(0.2)$ & $9.3(0.4)$ & $9.4(0.2)$ & $26.3(1.6)$ & $27.6(0.7)$ & $26.8(1.8)$ & $27.7(0.7)$ & 100 & 100 & 100 & 100 \\
\hline Heart Failure & $6.2(0.3)$ & $5.5(0.1)$ & $5.8(0.4)$ & $5.6(0.1)$ & 100 & 100 & 100 & 100 & $10.4(1.0)$ & $8.5(0.4)$ & $9.8(1.1)$ & $8.6(0.4)$ \\
\hline Hypertension & $42.2(0.7)$ & $44.4(0.3)$ & $43.7(0.8)$ & $44.2(0.3)$ & $59.7(1.8)$ & $62.1(0.8)$ & $59.9(2.0)$ & $61.9(0.8)$ & $45.7(1.7)$ & $44.2(0.6)$ & $47.2(1.8)$ & $44.0(0.7)$ \\
\hline Ischemic Heart Disease & $25.7(0.6)$ & $24.6(0.3)$ & $25.1(0.7)$ & $24.8(0.3)$ & $70.9(1.6)$ & $68.9(0.7)$ & $71.2(1.8)$ & $69.1(0.7)$ & $31.3(1.6)$ & $30.5(0.6)$ & $30.6(1.7)$ & $30.7(0.6)$ \\
\hline Arthritis & $36.7(0.7)$ & $37.2(0.3)$ & $37.9(0.8)$ & $36.9(0.3)$ & $41.2(1.8)$ & $43.0(0.8)$ & $41.9(2.0)$ & $42.7(0.8)$ & $44.4(1.7)$ & $45.2(0.7)$ & $45.6(1.8)$ & $44.9(0.7)$ \\
\hline Anxiety disorders and depression & $6.5(0.4)$ & $8.1(0.2)$ & $6.9(0.4)$ & $8.0(0.2)$ & $8.8(1.0)$ & $11.0(0.5)$ & $10.3(1.3)$ & $10.9(0.5)$ & $11.8(1.1)$ & $13.0(0.4)$ & $12.0(1.2)$ & $12.9(0.4)$ \\
\hline Schizophrenia & $0.3(0.1)$ & $0.3(0.0)$ & $0.3(0.1)$ & $0.3(0.0)$ & $0.7(0.3)$ & $0.2(0.1)$ & $0.7(0.3)$ & $0.3(0.1)$ & $0.2(0.2)$ & $0.2(0.1)$ & $0.3(0.2)$ & $0.2(0.1)$ \\
\hline Asthma & $3.5(0.3)$ & $3.4(0.1)$ & $3.6(0.3)$ & $3.4(0.1)$ & $7.2(0.9)$ & $8.0(0.4)$ & $6.9(1.0)$ & $7.9(0.4)$ & $23.9(1.4)$ & $29.3(0.6)$ & $24.7(1.6)$ & $29.1(0.6)$ \\
\hline \multicolumn{13}{|l|}{ Resource Utilization Band (RUB) } \\
\hline No use of health care services & $1.0(0.1)$ & $0.9(0.1)$ & $0.9(0.1)$ & $0.9(0.1)$ & $1.1(0.4)$ & $1.0(0.2)$ & $0.7(0.3)$ & $1.1(0.2)$ & $1.7(0.4)$ & $0.8(0.1)$ & $1.4(0.4)$ & $0.8(0.1)$ \\
\hline Healthy user of services & $0.8(0.1)$ & $0.5(0.0)$ & $0.7(0.1)$ & $0.5(0.0)$ & $0.4(0.2)$ & $0.6(0.1)$ & $0.4(0.2)$ & $0.6(0.1)$ & $1.2(0.4)$ & $1.0(0.1)$ & $1.0(0.3)$ & $1.0(0.1)$ \\
\hline Light morbidity & $16.7(0.5)$ & $15.6(0.2)$ & $17.1(0.6)$ & $15.7(0.2)$ & $4.1(0.7)$ & $3.6(0.3)$ & $3.4(0.7)$ & $3.6(0.3)$ & $6.7(0.8)$ & $5.0(0.3)$ & $6.5(0.9)$ & $5.1(0.3)$ \\
\hline Moderate morbidity & $58.7(0.7)$ & $61.0(0.3)$ & $59.0(0.8)$ & $60.8(0.3)$ & $55.8(1.8)$ & $53.9(0.8)$ & $57.3(2.0)$ & $53.9(0.8)$ & $56.7(1.7)$ & $58.6(0.6)$ & $57.2(1.8)$ & $58.5(0.6)$ \\
\hline
\end{tabular}

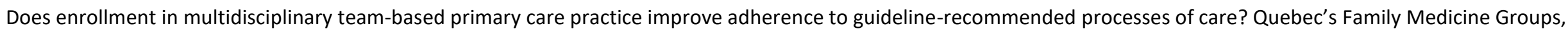
2002-2010 M Diop, J Fiset-Laniel, S Provost, P Tousignant, RB Da Silva, MJ Ouimet. Health Policy 121 (4), $378-388$ 


\begin{tabular}{|c|c|c|c|c|c|c|c|c|c|c|c|c|}
\hline & \multicolumn{4}{|c|}{ Diabetes $65+$} & \multicolumn{4}{|c|}{ Heart failure $65+$} & \multicolumn{4}{|c|}{ COPD de $65+$} \\
\hline & \multicolumn{2}{|c|}{ Unweighted } & \multicolumn{2}{|c|}{ Weighted } & \multicolumn{2}{|c|}{ Unweighted } & \multicolumn{2}{|c|}{ Weighted } & \multicolumn{2}{|c|}{ Unweighted } & \multicolumn{2}{|c|}{ Weighted } \\
\hline & FMG & non-FMG & FMG & non-FMG & FMG & non-FMG & FMG & non-FMG & FMG & non-FMG & FMG & non-FMG \\
\hline & $(n=5,069)$ & $(n=30,019)$ & $(n=5,069)$ & $(n=30,019)$ & $(n=764)$ & $(n=4,164)$ & $(n=764)$ & $(n=4,164)$ & $(n=896)$ & $(n=5,936)$ & $(n=896)$ & $(n=5,936)$ \\
\hline & $\%(S E)$ & $\%(S E)$ & $\%(S E)$ & $\%(S E)$ & $\%(S E)$ & $\%(S E)$ & $\%(S E)$ & $\%(S E)$ & $\%(S E)$ & $\%(S E)$ & $\%(S E)$ & $\%(S E)$ \\
\hline High morbidity & $15.0(0.5)$ & $14.0(0.2)$ & $15.1(0.6)$ & $14.0(0.2)$ & $23.6(1.5)$ & $24.1(0.7)$ & $23.6(1.7)$ & $23.9(0.7)$ & $21.2(1.4)$ & $22.1(0.5)$ & $22.1(1.5)$ & $22.1(0.5)$ \\
\hline Very high morbidity & $7.8(0.4)$ & $8.1(0.2)$ & $7.3(0.4)$ & $8.1(0.2)$ & $15.2(1.3)$ & $16.9(0.6)$ & $14.6(1.4)$ & $17.0(0.6)$ & $12.5(1.1)$ & $12.5(0.4)$ & $11.9(1.1)$ & $12.6(0.4)$ \\
\hline \multicolumn{13}{|c|}{ Tertiary Health Service Utilization-mean } \\
\hline Number of Emergency Room visits & $0.7(0.0)$ & $0.6(0.0)$ & $0.6(0.0)$ & $0.6(0.0)$ & $1.2(0.1)$ & $1.1(0.0)$ & $1.2(0.1)$ & $1.1(0.0)$ & $1.2(0.1)$ & $1.2(0.0)$ & $1.2(0.1)$ & $1.2(0.0)$ \\
\hline $\begin{array}{l}\text { Number of ER visits for ambulatory } \\
\text { care sensitive conditions }\end{array}$ & $0.0(0.0)$ & $0.0(0.0)$ & $0.0(0.0)$ & $0.0(0.0)$ & $0.0(0.0)$ & $0.0(0.0)$ & $0.0(0.0)$ & $0.0(0.0)$ & $0.0(0.0)$ & $0.0(0.0)$ & $0.0(0.0)$ & $0.0(0.0)$ \\
\hline $\begin{array}{l}\text { Number of ER visits with } \\
\text { hospitalization }\end{array}$ & $0.1(0.0)$ & $0.1(0.0)$ & $0.1(0.0)$ & $0.1(0.0)$ & $0.2(0.0)$ & $0.2(0.0)$ & $0.2(0.0)$ & $0.2(0.0)$ & $0.2(0.0)$ & $0.2(0.0)$ & $0.2(0.0)$ & $0.2(0.0)$ \\
\hline Number of hospitalizations & $0.2(0.0)$ & $0.2(0.0)$ & $0.2(0.0)$ & $0.2(0.0)$ & $0.4(0.0)$ & $0.4(0.0)$ & $0.4(0.0)$ & $0.4(0.0)$ & $0.4(0.0)$ & $0.4(0.0)$ & $0.4(0.0)$ & $0.4(0.0)$ \\
\hline $\begin{array}{l}\text { Number of hospitalizations for } \\
\text { ambulatory care sensitive conditions }\end{array}$ & $0.0(0.0)$ & $0.0(0.0)$ & $0.0(0.0)$ & $0.0(0.0)$ & $0.1(0.0)$ & $0.1(0.0)$ & $0.1(0.0)$ & $0.1(0.0)$ & $0.1(0.0)$ & $0.1(0.0)$ & $0.1(0.0)$ & $0.1(0.0)$ \\
\hline \multicolumn{13}{|c|}{ Ambulatory Health Service Utilization -mean } \\
\hline $\begin{array}{l}\text { Number of ambulatory consultations } \\
\text { (All) }\end{array}$ & \begin{tabular}{|c|}
10.1( \\
$0.1)$
\end{tabular} & $11.1(0.1)$ & $10.7(0.2)$ & $11.0(0.1)$ & $12.7(0.3)$ & $14.4(0.2)$ & $14.1(0.5)$ & $14.2(0.2)$ & $11.9(0.3)$ & $13.3(0.1)$ & $12.9(0.3)$ & $13.2(0.1)$ \\
\hline Number of consultations (Generalist) & $5.4(0.1)$ & $5.9(0.0)$ & $5.8(0.1)$ & $5.8(0.0)$ & $6.5(0.2)$ & $7.4(0.1)$ & $7.2(0.3)$ & $7.3(0.1)$ & $6.9(0.2)$ & $7.6(0.1)$ & $7.5(0.2)$ & $7.5(0.1)$ \\
\hline Number of consultations (Specialist) & $4.6(0.1)$ & $5.2(0.0)$ & $5.0(0.1)$ & $5.1(0.0)$ & $6.2(0.3)$ & $7.0(0.1)$ & $6.9(0.3)$ & $6.8(0.1)$ & $5.1(0.2)$ & $5.8(0.1)$ & $5.4(0.2)$ & $5.7(0.1)$ \\
\hline Number of different physicians seen & $4.8(0.1)$ & $5.1(0.0)$ & $4.9(0.1)$ & $5.0(0.0)$ & $6.1(0.2)$ & $6.5(0.1)$ & $6.5(0.2)$ & $6.4(0.1)$ & $5.8(0.1)$ & $6.2(0.1)$ & $6.0(0.2)$ & $6.1(0.1)$ \\
\hline Number of different generalists seen & $2.2(0.0)$ & $2.1(0.0)$ & $2.2(0.0)$ & $2.1(0.0)$ & $2.5(0.1)$ & $2.6(0.0)$ & $2.6(0.1)$ & $2.6(0.0)$ & $2.8(0.1)$ & $2.9(0.0)$ & $2.9(0.1)$ & $2.9(0.0)$ \\
\hline Number of different specialists seen & $2.7(0.0)$ & $2.9(0.0)$ & $2.8(0.0)$ & $2.9(0.0)$ & $3.6(0.1)$ & $3.8(0.1)$ & $3.9(0.2)$ & $3.8(0.1)$ & $3.0(0.1)$ & $3.3(0.0)$ & $3.1(0.1)$ & $3.3(0.0)$ \\
\hline \multicolumn{13}{|l|}{ Usual providers of care (UPC) } \\
\hline No UPC - $($ UPC < 0.75$)$ & $17.1(0.5)$ & $17.6(0.2)$ & $15.5(0.5)$ & $17.8(0.2)$ & $19.0(1.4)$ & $17.1(0.6)$ & $18.0(1.5)$ & $17.4(0.6)$ & $25.0(1.5)$ & $24.3(0.6)$ & $23.8(1.5)$ & $24.7(0.6)$ \\
\hline With UPC $-($ UPC $>=0.75)$ & $75.3(0.6)$ & $77.8(0.2)$ & $79.4(0.6)$ & $77.1(0.3)$ & $67.3(1.7)$ & $75.0(0.7)$ & $72.5(1.7)$ & $74.0(0.7)$ & $63.4(1.6)$ & $69.6(0.6)$ & $68.6(1.6)$ & $68.6(0.6)$ \\
\hline $\begin{array}{l}\text { Low users - (two or less visits during } \\
\text { last two years) }\end{array}$ & $7.6(0.4)$ & $4.6(0.1)$ & $5.1(0.3)$ & $5.1(0.1)$ & $13.7(1.3)$ & $7.9(0.4)$ & $9.5(1.0)$ & $8.6(0.5)$ & $11.6(1.1)$ & $6.2(0.3)$ & $7.7(0.8)$ & $6.7(0.3)$ \\
\hline \multicolumn{13}{|l|}{ Additional coverage RAMQ } \\
\hline yes & $95.5(0.3)$ & $94.9(0.1)$ & $93.8(0.4)$ & $95.1(0.1)$ & $97.1(0.6)$ & $96.4(0.3)$ & $95.6(1.0)$ & $96.5(0.3)$ & 100 & 100 & 100 & 100 \\
\hline \multicolumn{13}{|c|}{ Physician paid via salary (CLSC or UMF) } \\
\hline yes & | $16.0(0.5)$ & $9.7(0.2)$ & $15.6(0.6)$ & $9.8(0.2)$ & $20.3(1.5)$ & $12.4(0.5)$ & $19.2(1.6)$ & $12.9(0.5)$ & $18.0(1.3)$ & $10.8(0.4)$ & $16.0(1.3)$ & $11.0(0.4)$ \\
\hline \multicolumn{13}{|l|}{ Outcomes } \\
\hline $\begin{array}{l}\text { Diabetes-At least one prescription } \\
\text { IECA or ARA over two years }\end{array}$ & $72.8(0.6)$ & $69.2(0.3)$ & $69.6(0.7)$ & $69.7(0.3)$ & $91.7(2.0)$ & $86.7(1.1)$ & $88.8(2.8)$ & $87.3(1.0)$ & $75.2(4.1)$ & $75.1(1.7)$ & $73.6(4.6)$ & $75.6(1.7)$ \\
\hline
\end{tabular}

Does enrollment in multidisciplinary team-based primary care practice improve adherence to guideline-recommended processes of care? Quebec's Family Medicine Groups, 2002-2010 M Diop, J Fiset-Laniel, S Provost, P Tousignant, RB Da Silva, MJ Ouimet. Health Policy 121 (4), 378-388 


\begin{tabular}{|c|c|c|c|c|c|c|c|c|c|c|c|c|}
\hline & \multicolumn{4}{|c|}{ Diabetes $65+$} & \multicolumn{4}{|c|}{ Heart failure $65+$} & \multicolumn{4}{|c|}{ COPD de $65+$} \\
\hline & \multicolumn{2}{|c|}{ Unweighted } & \multicolumn{2}{|c|}{ Weighted } & \multicolumn{2}{|c|}{ Unweighted } & \multicolumn{2}{|c|}{ Weighted } & \multicolumn{2}{|c|}{ Unweighted } & \multicolumn{2}{|c|}{ Weighted } \\
\hline & FMG & non-FMG & FMG & non-FMG & FMG & non-FMG & FMG & non-FMG & FMG & non-FMG & FMG & non-FMG \\
\hline & $(n=5,069)$ & $(n=30,019)$ & $(n=5,069)$ & $(n=30,019)$ & $(n=764)$ & $(n=4,164)$ & $(n=764)$ & $(n=4,164)$ & $(n=896)$ & $(n=5,936)$ & $(n=896)$ & $(n=5,936)$ \\
\hline & $\%(S E)$ & $\%(S E)$ & $\%(S E)$ & $\%(S E)$ & $\%(S E)$ & $\%(S E)$ & $\%(S E)$ & $\%(S E)$ & $\%(S E)$ & $\%(S E)$ & $\%(S E)$ & $\%(S E)$ \\
\hline $\begin{array}{l}\text { Diabetes-At least one prescription } \\
\text { Statin over two years }\end{array}$ & $58.9(0.7)$ & $53.4(0.3)$ & $54.8(0.8)$ & $54.3(0.3)$ & $70.5(3.3)$ & $62.0(1.5)$ & $67.0(3.9)$ & $62.9(1.5)$ & $57.5(4.7)$ & $52.9(1.9)$ & $55.4(5.1)$ & $53.6(1.9)$ \\
\hline $\begin{array}{l}\text { Diabetes- At least one } \\
\text { ophthalmologist or optometrist } \\
\text { consultation over two years }\end{array}$ & $84.7(0.5)$ & $84.3(0.2)$ & $83.9(0.6)$ & $84.4(0.2)$ & $83.4(2.7)$ & $82.5(1.2)$ & $83.8(2.9)$ & $82.5(1.2)$ & $77.9(3.9)$ & $85.3(1.4)$ & $77.2(4.37)$ & $85.4(1.4)$ \\
\hline $\begin{array}{l}\text { Heart failure -At least one } \\
\text { prescription IECA or ARA over two } \\
\text { years }\end{array}$ & $76.2(3.1)$ & $72.6(1.4)$ & $72.5(3.7)$ & $73.1(1.4)$ & $65.4(1.7)$ & $61.5(0.8)$ & $61.2(2.0)$ & $62.0(0.8)$ & $51.5(6.2)$ & $48.0(2.8)$ & $43.2(6.8)$ & $48.3(2.8)$ \\
\hline $\begin{array}{l}\text { Heart failure -At least one GP or } \\
\text { cardiologist consultation over two } \\
\text { years }\end{array}$ & $96.4(1.3)$ & $98.8(0.3)$ & $97.8(0.8)$ & $98.7(0.4)$ & $97.0(0.6)$ & $99.1(0.1)$ & $98.3(0.4)$ & $99.0(0.2)$ & $97.0(2.1)$ & $98.8(0.6)$ & $98.3(1.3)$ & $98.6(0.7)$ \\
\hline $\begin{array}{l}\text { COPD-Patients with prescription } \\
\text { steroid- At least one prescription } \\
\text { bronchodilator over two year }\end{array}$ & $98.2(1.2)$ & $97.2(0.6)$ & $97.7(1.8)$ & $97.2(0.6)$ & 100 & $96.3(1.0)$ & 100 & $96.6(1.0)$ & $97.7(0.5)$ & $96.0(0.3)$ & $96.6(0.8)$ & $96.2(0.3)$ \\
\hline
\end{tabular}


Table 3: Predicted adherence to disease-specific guidelines before and after enrollment, and the estimated effects of enrollment in a FMG on guideline adherence

\begin{tabular}{|c|c|c|c|c|c|c|c|c|c|c|c|c|}
\hline \multirow{3}{*}{ Outcomes } & \multicolumn{4}{|c|}{ FMG } & \multicolumn{4}{|c|}{ Non-FMG } & \multirow{2}{*}{\multicolumn{2}{|c|}{$\mathrm{DD}$}} & \multirow{2}{*}{\multicolumn{2}{|c|}{$\begin{array}{l}\text { DD estimate as \% of } \\
\text { pre-period average }\end{array}$}} \\
\hline & \multicolumn{2}{|r|}{ Pre } & \multicolumn{2}{|r|}{ Post } & \multicolumn{2}{|r|}{ Pre } & \multicolumn{2}{|r|}{ Post } & & & & \\
\hline & $\%$ & $(95 \% \mathrm{Cl})$ & $\%$ & $(95 \% \mathrm{Cl})$ & $\%$ & $(95 \% \mathrm{Cl})$ & $\%$ & $(95 \% \mathrm{Cl})$ & $\%$ Points & $(95 \% \mathrm{Cl})$ & $\%$ & $(95 \% \mathrm{Cl})$ \\
\hline \multicolumn{13}{|c|}{ Diabetes $(\mathrm{N}=35088, \mathrm{FMG}=5,069$ and Non-FMG $=30,019$ ) } \\
\hline $\begin{array}{l}\text { At least one ACEI or ARB prescription } \\
\text { over two years } \\
\text { At least one Statin prescription over } \\
\text { two years } \\
\text { At least one ophthalmologist or } \\
\text { optometrist consultation over two years }\end{array}$ & $\begin{array}{l}73.3 \\
62.0 \\
83.4\end{array}$ & $\begin{array}{l}(72.1 ; 74.5) \\
(60.7 ; 63.4) \\
(82.2 ; 84.6)\end{array}$ & $\begin{array}{l}74.5 \\
63.8 \\
84.5\end{array}$ & $\begin{array}{l}(73.3 ; 75.7) \\
(62.4 ; 65.1) \\
(83.7 ; 85.3)\end{array}$ & $\begin{array}{l}73.3 \\
63.1 \\
84.1\end{array}$ & $\begin{array}{l}(72.3 ; 74.3) \\
(61.9 ; 64.2) \\
(82.8 ; 85.3)\end{array}$ & $\begin{array}{l}76.9 \\
67.0 \\
84.1\end{array}$ & $\begin{array}{l}(76.5 ; 77.3) \\
(66.5 ; 67.4) \\
(83.8 ; 84.4)\end{array}$ & $\begin{array}{c}-2.36^{\star \star *} \\
-2.19^{* \star *} \\
1.08\end{array}$ & $\begin{array}{l}(-3.46 ;-1.27) \\
(-3.42 ;-0.96) \\
(-0.38 ; 2.54)\end{array}$ & $\begin{array}{l}-3.39 \\
-4.04 \\
1.28\end{array}$ & $\begin{array}{l}(-4.97 ;-1.82) \\
(-6.31 ;-1.77) \\
(-0.45 ; 3.01)\end{array}$ \\
\hline \multicolumn{13}{|c|}{ Heart failure ( $N=4,928 \mathrm{FMG}=764$ and Non-FMG=4,164) } \\
\hline $\begin{array}{l}\text { At least one ACEI combination with a } \\
\text { beta- blocker or ARB prescription over } \\
\text { two years }\end{array}$ & 61.9 & $(58.2 ; 65.5)$ & 57.2 & $(53.7 ; 60.6)$ & 64.9 & $(61.8 ; 68.1)$ & 60.8 & $(59.5 ; 62.2)$ & -0.61 & $(-4.11 ; 2.89)$ & -0.98 & $(-6.62 ; 4.65)$ \\
\hline $\begin{array}{l}\text { At least one bronchodilator prescription } \\
\text { over two years among patients with } \\
\text { steroid prescription }\end{array}$ & 96.5 & $(95.0 ; 98.1)$ & 96.9 & $(95.7 ; 98.1)$ & 96.0 & $(94.7 ; 97.4)$ & 96.3 & $(95.8 ; 96.7)$ & 0.10 & $(-1.23 ; 1.43)$ & 0.10 & $(-1.28 ; 1,49)$ \\
\hline
\end{tabular}

${ }^{*}=p$-value $<.05 ;{ }^{* *}=p$-value $<.01 ; * *=p$-value $<.001$

All values reflect IPTW adjustment. More detail on the outcome indicators is included in Appendix 4. 


\section{Adherence to disease-specific guidelines}

The rate of diabetes-related guideline adherence after patient enrollment increased in both FMG and non-FMG practices, particularly for prescription drug-related guidelines (Table 3 ). Because these increases are larger among non-FMG patients, the estimated effects of FMGs on adherence to prescription of ACE inhibitors or ARBs and prescription of statins are -2.36 and -2.19 percentage points, respectively. These represent an average relative reduction in adherence of $3.39 \%$ and $4.04 \%$, respectively. Both groups improve over time, but adherence among the FMG patients improves less. We do not find evidence of an impact of enrollment in an FMG on adherence to recommended consultations with an optometrist or an ophthalmologist (DD [95\% CI] $1.08[-0.38 ; 2.54])$.

The percentage of patients with HF who have at least one prescription for ACE inhibitors or ARBs in the last two years decreased among FMG and non-FMG patients while the percentage who had at least one consultation with a general practitioner or a cardiologist in the past two years rose in both groups. We find no effect of FMG enrollment on rates of guideline adherence in HF patients: prescription DD [95\% $\mathrm{Cl}]=-0.61[-4.11 ; 2.89]$ and consultation $\mathrm{DD}[95 \% \mathrm{Cl}]=-2.70[-11.34 ; 5.94]$.

Adherence to guidelines for prescription bronchodilators for patients with COPD and taking steroids was already very high. Table 3 shows little change in adherence after enrollment among either FMG or non-FMG patients. We found no evidence of an effect of FMG on adherence to guidelines in patients with COPD (DD [95\% Cl] $0.29[-1.19 ; 1.77])$.

\section{Adherence to composite guidelines}

The proportion of patients who filled prescriptions for all or any guideline-recommended drugs increased over time in both groups, though the growth was greater among non-FMG patients (Table 4).

Does enrollment in multidisciplinary team-based primary care practice improve adherence to guidelinerecommended processes of care? Quebec's Family Medicine Groups, 2002-2010 M Diop, J Fiset-Laniel, S Provost, P Tousignant, RB Da Silva, MJ Ouimet. Health Policy 121 (4), 378-388 
Table 4: Predicted adherence to composite guidelines before and after enrollment, and the estimated effects of enrollment in a FMG on guideline adherence

\begin{tabular}{|c|c|c|c|c|c|c|c|c|c|c|c|c|}
\hline \multirow{3}{*}{ Outcomes } & \multicolumn{4}{|c|}{ FMG } & \multicolumn{4}{|c|}{ Non-FMG } & \multirow{2}{*}{\multicolumn{2}{|c|}{$\mathrm{DD}$}} & \multirow{2}{*}{\multicolumn{2}{|c|}{$\begin{array}{l}\mathrm{DD} \text { estimate as } \% \text { of } \\
\text { pre-period average }\end{array}$}} \\
\hline & \multicolumn{2}{|r|}{ Pre } & \multicolumn{2}{|r|}{ Post } & \multicolumn{2}{|r|}{ Pre } & \multicolumn{2}{|r|}{ Post } & & & & \\
\hline & $\%$ & $(95 \% \mathrm{Cl})$ & $\%$ & $(95 \% \mathrm{Cl})$ & $\%$ & $(95 \% \mathrm{Cl})$ & $\%$ & $(95 \% \mathrm{Cl})$ & $\%$ Points & $(95 \% \mathrm{Cl})$ & $\%$ & $(95 \% \mathrm{Cl})$ \\
\hline \multicolumn{13}{|c|}{ Drugs ( $N=44,599$ FMG=6,376 and Non-FMG=38,223) } \\
\hline Adherence to $100 \%$ of guidelines & 55.0 & $(53.7 ; 56.3)$ & 55.6 & $(54.5 ; 56.8)$ & 54.9 & $(53.8 ; 56.0)$ & 58.4 & $(57.9 ; 58.8)$ & $-2.83^{* * *}$ & $(-4.08 ;-1.58)$ & -5.74 & $(-8.27 ;-3.20)$ \\
\hline Adherence to $75 \%$ of guidelines & 55.3 & $(54.0 ; 56.5)$ & 55.9 & $(54.7 ; 57.1)$ & 55.2 & $(54.1 ; 56.3)$ & 58.8 & $(58.3 ; 59.2)$ & $-2.92^{\star * *}$ & $(-4.17 ;-1.67)$ & -3.63 & $(-5.18 ;-2.08)$ \\
\hline Adherence to $50 \%$ of guidelines & 81.7 & $(80.8 ; 82.7)$ & 82.9 & $(82.1 ; 83.8)$ & 81.4 & $(80.6 ; 82.3)$ & 84.6 & $(84.3 ; 84.9)$ & $-1.93^{* * *}$ & $(-2.85 ;-1.01)$ & -3.91 & $(-5.77 ;-2.04)$ \\
\hline Adherence to at least one guideline & 83.9 & $(83.0 ; 84.8)$ & 85.0 & $(84.2 ; 85.8)$ & 83.8 & $(83.0 ; 84.6)$ & 86.6 & $(86.3 ; 86.9)$ & $-1.72^{* * *}$ & $(-2.57 ;-0.87)$ & -2.09 & $(-3.13 ;-1.06)$ \\
\hline \multicolumn{13}{|c|}{ Visits ( $N=36,336$ FMG $=4,994$ and Non-FMG=31,342) } \\
\hline Adherence to $100 \%$ of guidelines & 82.9 & $(81.6 ; 84.1)$ & 84.1 & $(83.3 ; 84.9)$ & 82.6 & $(80.7 ; 84.4)$ & 84.0 & $(83.6 ; 84.5)$ & -0.24 & $(-2.24 ; 1.75)$ & -0.29 & $(-2.66 ; 2.08)$ \\
\hline Adherence to at least one guideline & 87.0 & $(85.6 ; 88.4)$ & 88.1 & $(86.9 ; 89.3)$ & 87.3 & $(86.2 ; 88.5)$ & 87.9 & $(87.6 ; 88.3)$ & 0.49 & $(-0.81 ; 1.78)$ & 0.56 & $(-0.93 ; 2.05)$ \\
\hline \multicolumn{13}{|c|}{ Drugs and visits ( $\mathrm{N}=41,465 \mathrm{FMG}=5,605$ and Non-FMG=35,860) } \\
\hline Adherence to $100 \%$ of guidelines & 50.3 & $(47.9 ; 52.8)$ & 51.4 & $(49.3 ; 53.4)$ & 48.7 & $(47.0 ; 50.4)$ & 53.3 & $(52.9 ; 53.8$ & $-3.57^{\star * *}$ & $(-5.70 ;-1.44)$ & -7.83 & $(-12.49 ;-3.16)$ \\
\hline Adherence to $75 \%$ of guidelines & 52.8 & $(50.4 ; 55.3)$ & 53.9 & $(51.9 ; 55.9)$ & 51.5 & $(49.8 ; 53.2)$ & 56.6 & $(56.2 ; 57.1)$ & $-4.03^{* * *}$ & $(-6.20 ;-1.85)$ & -8.59 & $(-13.21 ;-3.94)$ \\
\hline Adherence to $50 \%$ of guidelines & 80.9 & $(79.8 ; 82.1)$ & 82.5 & $(81.5 ; 83.5)$ & 80.5 & $(78.7 ; 82.3)$ & 84.5 & $(84.2 ; 84.9)$ & $-2.50^{* *}$ & $(-4.36 ;-0.65)$ & -3.17 & $(-5.53 ;-0.82)$ \\
\hline Adherence to at least one guideline & 97.1 & $(96.5 ; 97.7)$ & 97.8 & $(97.4 ; 98.2)$ & 97.2 & $(96.6 ; 97.8)$ & 97.8 & $(97.7 ; 97.9)$ & 0.13 & $(-0.56 ; 0.81)$ & 0.13 & $(-0.58 ; 0.84)$ \\
\hline
\end{tabular}

${ }^{*}=p$-value $<.05 ;{ }^{* *}=p$-value $<.01 ;{ }^{* * *}=p$-value $<.001$

All values reflect IPTW adjustment. More detail on the composite measures is included in Appendix 4. 
The DD estimates indicate a relative decrease in adherence for FMG patients: FMG enrollment led to a reduction of $5.74 \%$ reduction in the probability of adherence to all prescription drug-related guidelines and a $2.09 \%$ reduction in the probability of adherence to at least one prescription drug-related guideline.

Small increases in rates of adherence to consultation-related guidelines are observed among both FMG patients and non-FMG patients. The percentage of patients who follow all recommendations increases from $82.9 \%$ to $84.1 \%$ for FMG patients and $82.6 \%$ to $84.0 \%$ for others. We find no evidence of an effect of FMG enrollment on consultation-related guideline adherence.

\section{DISCUSSION}

After five years of follow-up, a reasonable length of time to detect impacts of primary care reforms on process measures of quality of care, we find no evidence of a positive impact of FMG enrollment on rates of guideline adherence for the chronic diseases considered. In fact, both FMG and non-FMG patients show slight, and in almost all cases statistically indistinguishable, improvements in adherence to guidelines after enrollment with their physician. We did find a negative impact of FMGs on adherence to prescription drug-related guidelines, particularly for patients with diabetes. Given the similarities and differences between FMGs and other team-based primary care reform models, these results add to the growing body of evidence regarding what does, and does not, "work" in Quebec and in other jurisdictions.

Based on the multidisciplinary, team-based design of FMGs and the existing literature showing higher rates of guideline-recommended care among such practices $(26,28-31)$, we were somewhat surprised by our finding of no positive effects of FMGs. Several factors may contribute to explaining these results. In part, a policy that was well-designed but not well-implemented may mean that the expected changes in the organization of care were not fully realized. Recent evidence suggests that many practices did not

Does enrollment in multidisciplinary team-based primary care practice improve adherence to guidelinerecommended processes of care? Quebec's Family Medicine Groups, 2002-2010 M Diop, J Fiset-Laniel, S Provost, P Tousignant, RB Da Silva, MJ Ouimet. Health Policy 121 (4), 378-388 
fulfill their responsibilities vis-à-vis improving access(50) and relatively little is known about how or if the different health professionals collaborate in FMG practices. Second, organizational structure and provider payment mechanisms potentially interact in important ways. Reforms that do not include both may be less effective at changing clinical practice. Relative to effects seen in other multidisciplinary, team-based primary care models, the fact that the FMG policy does not include any pay-for-performance incentives or capitation-based payment may contribute to the null results we found. Third, all patients in our study were enrolled with a GP, and this enrollment may lead to GPs taking more responsibility for their enrolled patients and increased guideline adherence regardless of the organizational model. Since both FMG and non-FMG patients in our data are enrolled with a GP, we are not able to test any hypotheses regarding the impacts of enrollment.

While the negative effect of FMGs on rates of adherence to prescription drug-related guidelines was contrary to our a priori hypothesis, there are alternative interpretations beyond that enrolling in an FMG results in lower-quality care. Clinical guidelines evolve over time and they are sometimes questioned, especially regarding medications(65). Some argue that guidelines may complicate care for patients with comorbidities, usually due to contraindications(66), and that guideline recommendations are not always appropriate for all patients. This negative FMG effect may also be related to the organizational characteristics of FMGs that could contribute to better tailoring of clinical recommendations to individual patients. The computerization of practices and the implementation of electronic medical records generally occurred earlier in FMG practices. Better availability of information on drugs in the patient record and alerts on drug interactions or contraindications could lead to a more judicious application of guidelines. The relative decrease in prescription-drug-related guideline adherence among FMG patients may also reflect more team-based case management, more frequent continuing education, or updates

Does enrollment in multidisciplinary team-based primary care practice improve adherence to guidelinerecommended processes of care? Quebec's Family Medicine Groups, 2002-2010 M Diop, J Fiset-Laniel, S Provost, P Tousignant, RB Da Silva, MJ Ouimet. Health Policy 121 (4), 378-388 
regarding clinical practice, if these are indeed more prevalent in FMG practices(67). Of course, our administrative health data limits our ability to distinguish between appropriate tailoring to patient needs and other reasons for not adhering to guidelines. However, the fact that we find no evidence of FMG effects on adherence to the less controversial consultation-related guidelines lends some support to the hypothesis that tailoring is occurring. Finally, we emphasize that our evaluation is fundamentally one of quantity, not quality. We estimate impacts of FMGs on rates of adherence to clinical guidelines, but such adherence is not in and of itself a sufficient measure to evaluate the quality of care $(68,69)$.

While this study contributes to the evidence base of what works, future research is needed to improve our understanding of why certain interventions do or do not achieve their objectives. We evaluated the early years of Quebec's primary care reform, which is ongoing and expanding, with substantial organizational heterogeneity both across teams and over time. An implementation science perspective could be useful in future work to understand the extent to which primary care reforms are implemented as intended, the nature of collaboration between physician and non-physician providers in team-based practices, how this has evolved over time, and how it is related to the achievement of desired outcomes. Evaluations of organizational reforms using data that could clarify whether guideline recommendations are tailored, or not applied, to patients with multiple chronic conditions could also illuminate the extent to which multidisciplinary team-based practices improve quality of care for these patients.

Quebec's FMGs have many structural similarities to other patient-centered medical home reforms, making our findings relevant and potentially generalizable to other health care systems. However, the FMG reform also differs in important ways, which allows us to examine the effects of multidisciplinary team-based primary health care on guideline-recommended care in the absence of financial incentives.

Does enrollment in multidisciplinary team-based primary care practice improve adherence to guidelinerecommended processes of care? Quebec's Family Medicine Groups, 2002-2010 M Diop, J Fiset-Laniel, S Provost, P Tousignant, RB Da Silva, MJ Ouimet. Health Policy 121 (4), 378-388 
We estimated the impacts of FMGs on adherence to clinical guidelines for three prevalent chronic conditions using rigorous empirical methods and a large, population-representative database. The DD method has the advantage of estimating the causal effects of the change in primary care organization, after controlling for fixed differences and time trends common to FMG and non-FMG patients. We use PS adjustment to make our treatment and control groups comparable at baseline, reducing the potential for confounding bias and making the assumptions of parallel pre-intervention outcome trends and no differential time-varying factors between the two groups more reasonable.

Our study also has some limitations. The data covered the first 79 of Quebec's FMGs, out of 258 FMG as of March 2014. The possibility of effect heterogeneity between early and later adopters remains an important question that should be addressed by future evaluations with more recent data. Due to the two-year reference period for each of our guidelines, we have only one pre-exposure data point. We are therefore limited to assessing whether the pre-exposure outcome levels, not trends, are similar among FMG and non-FMG patients. We also have a limited number of guidelines and measurable diseases in our databases. For example, it is not possible to measure recommendations related to physical exercise and healthy eating. Finally, the indicators related to drugs and consultations with an optometrist focus on those aged 65 and over, since only that group has universal public coverage for those services.

\section{CONCLUSIONS}

Evaluating the first seven years of Quebec's FMG primary care reform, we found no evidence of an effect of team-based FMGs on greater adherence to certain clinical guidelines for three prevalent chronic diseases. Future research is needed to assess why this example of team-based primary care practice did not improve performance on these quality-of-care indicators, and what elements of the implementation

Does enrollment in multidisciplinary team-based primary care practice improve adherence to guidelinerecommended processes of care? Quebec's Family Medicine Groups, 2002-2010 M Diop, J Fiset-Laniel, S Provost, P Tousignant, RB Da Silva, MJ Ouimet. Health Policy 121 (4), 378-388 
of the reform and the structure, organization, interaction, and resources of primary care teams contribute to this result.

Does enrollment in multidisciplinary team-based primary care practice improve adherence to guidelinerecommended processes of care? Quebec's Family Medicine Groups, 2002-2010 M Diop, J Fiset-Laniel, S Provost, P Tousignant, RB Da Silva, MJ Ouimet. Health Policy 121 (4), 378-388 


\section{References}

1. World Health Organization. WHO Global Forum on chronic diseases focuses attention on worldwide prevention efforts: World Health Organization; 2004 [cited 2015 March 25, 2015]. Available from: http://www.who.int/mediacentre/news/releases/2004/pr76/fr/.

2. World Health Organization. Noncommunicable diseases: World Health Organization; 2014 [cited 2015 March 23, 2015]. Cette page réunit les informations disponibles en français sur les maladies chroniques telles que le cancer, le diabète, les maladies respiratoires, l'asthme, et les pathologies cardiovasculaires.]. Available from: http://www.who.int/topics/chronic diseases/fr/.

3. Institut de la Statistique du Québec. Le bilan démographique du Québec. Institut de la Statistique du Québec, 2014.

4. Dubé F, Noreau É, Landry G. Cadre de référence pour la prévention et la gestion des maladies chroniques physiques en première ligne: Ministère de la santé et des services sociaux, Services de santé et médecine universitaire; 2012.

5. Arnold JMO, Liu P, Demers C, Dorian P, Giannetti N, Haddad H, et al. Canadian Cardiovascular Society consensus conference recommendations on heart failure 2006: Diagnosis and management. CJCA Canadian Journal of Cardiology. 2006;22(1):23-45.

6. McKelvie RS, Moe GW, Ezekowitz JA, Heckman GA, Costigan J, Ducharme A, et al. The 2012 Canadian Cardiovascular Society heart failure management guidelines update: focus on acute and chronic heart failure. The Canadian journal of cardiology. 2013;29(2):168-81.

7. O'Donnell DE, Hernandez P, Kaplan A, Aaron S, Bourbeau J, Marciniuk D, et al. Canadian Thoracic Society recommendations for management of chronic obstructive pulmonary disease-2008 updatehighlights for primary care. Canadian respiratory journal: journal of the Canadian Thoracic Society. 2008;15(Suppl A):1A.

8. Canadian Diabetes Association Clinical Practice Guidelines Expert Committee. Canadian Diabetes Association 2013 Clinical Practice Guidelines for the Prevention and Management of Diabetes in Canada. 2013 Contract No.: suppl 1.

9. Allen LA, O'Donnell CJ, Giugliano RP, Camargo CA, Lloyd-Jones DM. Care concordant with guidelines predicts decreased long-term mortality in patients with unstable angina pectoris and non-ST-elevation myocardial infarction. The American journal of cardiology. 2004;93(10):1218-22.

10. Kahn KL, Tisnado DM, Adams JL, Liu H, Chen WP, Hu FA, et al. Does Ambulatory Process of Care Predict Health-Related Quality of Life Outcomes for Patients with Chronic Disease? Health Serv Res. 2007;42(1p1):63-83.

11. Mangione CM. Preventing and managing chronic disease : Ontario's framework: Ministry of Health and Long-Term Care; 2007. Available from: http://hdl.handle.net/1873/13984.

12. Nazir SA, Erbland ML. Chronic obstructive pulmonary disease: an update on diagnosis and management issues in older adults. Drugs Aging. 2009;26(10):813-31.

13. Canadian Diabetes Association Clinical Practice Guidelines Expert Committee. Canadian Diabetes Association 2003 Clinical Practice Guidelines for the Prevention and Management of Diabetes in Canada. 2003 Contract No.: Suppl. 2.

14. Canadian Diabetes Association Clinical Practice Guidelines Expert Committee. Canadian Diabetes Association 2008 clinical practice guidelines for the prevention and management of diabetes in Canada. 2008 Contract No.: Suppl. 1.

15. Alan Kaplan M, Meyer Balter M, André Gervais M, Yves Lacasse M, Francois Maltais M, Graeme Rocker $\mathrm{M}$, et al. Recommandations de la Société canadienne de thoracologie au sujet de la prise en charge

Does enrollment in multidisciplinary team-based primary care practice improve adherence to guideline-recommended processes of care? Quebec's Family Medicine Groups, 2002-2010 M Diop, J Fiset-Laniel, S Provost, P Tousignant, RB Da Silva, MJ Ouimet. Health Policy 121 (4), 378-388 
de la maladie pulmonaire obstructive chronique-Mise à jour de 2008-Points saillants pour les soins primaires. Can Respir J. 2008;15:1A.

16. O'Donnell DE, Aaron S, Bourbeau J, Hernandez P, Marciniuk D, Balter M, et al. Canadian Thoracic Society recommendations for management of chronic obstructive pulmonary disease--2003. Canadian respiratory journal : journal of the Canadian Thoracic Society. 2003;10.

17. O'Donnell DE, Aaron S, Bourbeau J, Hernandez P, Marciniuk DD, Balter M, et al. Canadian Thoracic Society recommendations for management of chronic obstructive pulmonary disease - 2007 update. Canadian respiratory journal : journal of the Canadian Thoracic Society. 2007;14.

18. Russell GM, Dahrouge S, Hogg W, Geneau R, Muldoon L, Tuna M. Managing chronic disease in Ontario primary care: the impact of organizational factors. The Annals of Family Medicine. 2009;7(4):309-

18.

19. Pineault R, Provost S, Hamel M, Couture A, Levesque J. The influence of primary health care organizational models on patients' experience of care in different chronic disease situations. primary care. 2011;4:8.

20. Manns BJ, Tonelli M, Zhang J, Campbell DJ, Sargious P, Ayyalasomayajula B, et al. Enrolment in primary care networks: impact on outcomes and processes of care for patients with diabetes. Can Med Assoc J. 2011:cmaj. 110755.

21. Ashworth $M$, Armstrong $D$. The relationship between general practice characteristics and quality of care: a national survey of quality indicators used in the UK Quality and Outcomes Framework, 2004-5. BMC Family Practice. 2006;7(1):68.

22. Campbell S, Hann M, Hacker J, Burns C, Oliver D, Thapar A, et al. Identifying predictors of high quality care in English general practice: observational study. Bmj. 2001;323(7316):784.

23. Grumbach K, Bodenheimer T. Can health care teams improve primary care practice? JAMA. 2004;291(10):1246-51.

24. Gulliford MC, Jack RH, Adams G, Ukoumunne OC. Availability and structure of primary medical care services and population health and health care indicators in England. Bmc Health Serv Res. 2004;4(1):12.

25. Stevenson K, Baker R, Farooqi A, Sorrie R, Khunti K. Features of primary health care teams associated with successful quality improvement of diabetes care: a qualitative study. Fam Pract. 2001;18(1):21-6.

26. Fantini MP, Compagni A, Rucci P, Mimmi S, Longo F. General practitioners' adherence to evidencebased guidelines: a multilevel analysis. Health care management review. 2012;37(1):67-76.

27. Smith PC, York N. Quality incentives: the case of UK general practitioners. Health Aff (Millwood). 2004;23(3):112-8.

28. Li R, Simon J, Bodenheimer T, Gillies RR, Casalino L, Schmittdiel J, et al. Organizational factors affecting the adoption of diabetes care management processes in physician organizations. Diabetes Care. 2004;27(10):2312-6.

29. Scott A, Schurer S, Jensen PH, Sivey P. The effect of financial incentives on quality of care: The case of diabetes: Melbourne Institute of Applied Economic and Social Research, University of Melbourne; 2008.

30. Tu K, Cauch-Dudek K, Chen Z. Comparison of primary care physician payment models in the management of hypertension. Canadian Family Physician. 2009;55(7):719-27.

31. Bruni ML, Nobilio L, Ugolini C. Economic incentives in general practice: the impact of pay-forparticipation and pay-for-compliance programs on diabetes care. Health Policy. 2009;90(2):140-8.

32. Armour BS, Pitts MM, Maclean R, Cangialose C, Kishel M, Imai H, et al. The effect of explicit financial incentives on physician behavior. Arch Intern Med. 2001;161(10):1261-6.

Does enrollment in multidisciplinary team-based primary care practice improve adherence to guideline-recommended processes of care? Quebec's Family Medicine Groups, 2002-2010 M Diop, J Fiset-Laniel, S Provost, P Tousignant, RB Da Silva, MJ Ouimet. Health Policy 121 (4), 378-388 
33. Campbell SM, Reeves D, Kontopantelis E, Sibbald B, Roland M. Effects of pay for performance on the quality of primary care in England. New Engl J Med. 2009;361(4):368-78.

34. Christianson JB, Leatherman S, Sutherland K. Lessons from evaluations of purchaser pay-forperformance programs a review of the evidence. Medical Care Research and Review. 2008;65(6 suppl):5S$35 \mathrm{~S}$.

35. Giuffrida A, Gosden T, Forland F, Kristiansen I, Sergison M, Leese B, et al. Target payments in primary care: effects on professional practice and health care outcomes. The Cochrane Library. 1999.

36. Glazier RH, Klein-Geltink J, Kopp A, Sibley LM. Capitation and enhanced fee-for-service models for primary care reform: a population-based evaluation. Can Med Assoc J. 2009;180(11):E72-E81.

37. Petersen LA, Woodard LD, Urech T, Daw C, Sookanan S. Does pay-for-performance improve the quality of health care? Annals of internal medicine. 2006;145(4):265-72.

38. Rosenthal MB, Frank RG. What is the empirical basis for paying for quality in health care? Medical Care Research and Review. 2006;63(2):135-57.

39. Scott A, Sivey P, Ait Ouakrim D, Willenberg L, Naccarella L, Furler J, et al. The effect of financial incentives on the quality of health care provided by primary care physicians. The Cochrane Library. 2011.

40. Town R, Kane R, Johnson P, Butler M. Economic incentives and physicians' delivery of preventive care: a systematic review. American journal of preventive medicine. 2005;28(2):234-40.

41. Allen T, Mason T, Whittaker W. Impacts of pay for performance on the quality of primary care. Risk Management and Healthcare Policy. 2014;7:113-20.

42. Beaulieu M-D, Denis J-L, D'Amour D, Goudreau J, Haggerty J, Hudon É, et al. L'implantation des Groupes de médecine de famille: le défi de la réorganisation de la pratique et de la collaboration interprofessionnelle. Montréal, Chaire Docteur Sadok Besrour en médecine familiale. Montréal2006.

43. Ministère de la Santé et des Services sociaux. Devenir un GMF : Guide d'accompagnement - Le groupe de médecine de famille : un atout pour le patient et son médecin. les membres du groupe de soutien à l'implantation des groupes de médecine de famille, 2006 Contract No.: 23 septembre 2015. 44. Pomey M-P, Martin E, Forest P-G. Quebec's family medicine groups: innovation and compromise in the reform of front-line care. Canadian Political Science Review. 2009;3(4):31-46.

45. Ministère de la Santé et des Services sociaux. Document synthèse. In: La Direction générale des affaires médicales et universitaires editor. Québec: Ministère de la Santé et des Services sociaux; 2002.

46. Ministère de la Santé et des Services sociaux Devenir un GMF : Guide d'accompagnement - Le groupe de médecine de famille : un atout pour le patient et son médecin. In: La Direction générale des affaires médicales et universitaires -les membres du groupe de soutien à l'implantation des groupes de médecine de famille, editor. Québec: Ministère de la Santé et des Services sociaux; 2003.

47. Régie de l'assurance maladie du Québec. Entente particulière provisoire MAJ 2006 : relative à la prise en charge et au suivi des clientèles vulnérables entre le Ministère de la santé et des services sociaux et la fédération des médecins omnipraticiens du Québec Québec2006 [cited 2015 October 6, 2015]. Available from: http://www.ramq.gouv.qc.ca/SiteCollectionDocuments/professionnels/manuels/104-brochure-1omnipraticiens/007 enten particu entente omni.pdf.

48. Breton $M$, Lévesque J, Pineault R, Hogg W. L'implantation du modèle des groupes de médecine de famille au Québec: potentiel et limites pour l'accroissement de la performance des soins de santé primaires. Pratiques et organisation des soins. 2011;42(2):101-9.

49. Ministère de la Santé et des Services sociaux. Rapport annuel de gestion 2013-2014 Ministère de la Santé et des Services sociaux. Gouvernement du Québec, 2014 2014. Report No.

50. Vérificateur général du Québec. Vérification de l'optimisation des ressources. Rapport du Vérificateur général du Québec à l'Assemblée nationale pour l'année 2015-2016 2015.

Does enrollment in multidisciplinary team-based primary care practice improve adherence to guideline-recommended processes of care? Quebec's Family Medicine Groups, 2002-2010 M Diop, J Fiset-Laniel, S Provost, P Tousignant, RB Da Silva, MJ Ouimet. Health Policy 121 (4), 378-388 
51. Régie de l'assurance maladie du Québec. Présentation de la Régie de l'assurance maladie du Québec-Un partenaire dynamique dans la gestion et l'évolution du système de santé québécois,. Gouvernement du Québec, 2011.

52. Ministère de la Santé et des Services sociaux en collaboration avec l'Institut national de santé publique du Québec et l'Institut de la statistique du Québec. Pour guider l'action - Portrait de santé du Québec et de ses régions : les statistiques. gouvernement du Québec, 2011.

53. Pigeon É, Larocque I. Tendances temporelles de la prévalence et de l'incidence du diabète, et mortalité chez les diabétiques au Québec, de 2000-2001 à 2006-2007: Institut national de santé publique du Québec; 2011.

54. Lix L, Yogendran M, Burchill C, Metge C, McKeen N, Moore D, et al. Defining and validating chronic diseases: an administrative data approach. Citeseer, 20061896489257.

55. Gauthier J, Haggerty J. Entre adaptabilité et fragilité: les conditions d'accès aux services de santé des communautés rurales et éloignées: rapport de recherche: Direction des systèmes de soins et politiques publiques, Institut national de santé publique Québec; 2009.

56. Pampalon R, Hamel D, Gamache P, Raymond G. Un indice de défavorisation pour la planification de la santé au Canada. Maladies chroniques au Canada. 2009;29(4):199-213.

57. Austin PC. An introduction to propensity score methods for reducing the effects of confounding in observational studies. Multivariate behavioral research. 2011;46(3):399-424.

58. Oakes JM, Johnson PJ. Propensity score matching for social epidemiology. Methods in social epidemiology. 2006;1:370-93.

59. Coyle N, Strumpf E, Fiset-Laniel J, Tousignant P, Roy Y. Characteristics of physicians and patients who join team-based primary care practices: Evidence from Quebec's Family Medicine Groups. Health policy. 2014;116(2-3):264-72.

60. Hirano K, Imbens GW. Estimation of causal effects using propensity score weighting: An application to data on right heart catheterization. Health Services and Outcomes research methodology. 2001;2(34):259-78.

61. Fougère $D$. Les méthodes économétriques d'évaluation. Revue française des affaires sociales. 2010;1(1):105-28.

62. Strumpf E, Harper S, Kaufman J. Fixed Effects and Difference-in-Differences. In: Oakes JM, Kaufman JK, eds. Methods in Social Epidemiology. 2nd Edition ed. Jossey-Bass: John Wiley \& Sons. In Press. 2016.

63. Sevestre P. Econométrie des données de panel: Dunod Paris; 2002.

64. Dunkley-Hickin C. Effects of primary care reform in Quebec on access to primary health care services: McGill University; 2014.

65. Diamond DM, Ravnskov U. How statistical deception created the appearance that statins are safe and effective in primary and secondary prevention of cardiovascular disease. Expert review of clinical pharmacology. 2015;8(2):201-10.

66. van Weel C, Schellevis FG. Comorbidity and guidelines: conflicting interests. The Lancet. 2006;367(9510):550-1.

67. Pineault R, Da Silva RB, Prud'homme A, Fournier M, Couture A, Provost S, et al. Impact of Québec's healthcare reforms on the organization of primary healthcare (PHC): a 2003-2010 follow-up. BMC Health Services Research. 2014;14(1):229.

68. Stange KC. The paradox of the parts and the whole in understanding and improving general practice. International Journal for Quality in Health Care. 2002;14(4):267-8.

69. Stange KC, Ferrer RL. The paradox of primary care. The Annals of Family Medicine. 2009;7(4):293-9.

Does enrollment in multidisciplinary team-based primary care practice improve adherence to guideline-recommended processes of care? Quebec's Family Medicine Groups, 2002-2010 M Diop, J Fiset-Laniel, S Provost, P Tousignant, RB Da Silva, MJ Ouimet. Health Policy 121 (4), 378-388 


\section{Appendix 1: Variable definitions}

I. "Vulnerable" patients as defined for incentive payments for enrollment

A patient was considered vulnerable if he was either: (a) 70 years or older, or (b) suffering from any of the following health conditions (regardless of age): 1) psychosis, not including most depression; 2 ) chronic obstructive pulmonary disease (COPD), moderate to severe persistent asthma , occupational lung disease; 3 ) atherosclerotic heart disease; 4) cancer treated by systemic chemotherapy or radiotherapy, or in a palliative stage; 5) diabetes with target organ damage; 6) in recovery from hard drugs or alcohol addiction, in treatment with methadone; 7) HIV / AIDS: 8) degenerative diseases of the central nervous system; 9) inflammatory diseases: arthritis, rheumatoid arthritis, non-cutaneous psoriasis, lupus, scleroderma and other connective tissue disease, ulcerative colitis, Crohn's disease.

\section{The Pampalon deprivation index}

The Pampalon index is based on the level of education, employment, income and family structure (e.g. proportion of divorced or single-parent families) in a small spatial unit (census dissemination area) and is used by the Institut national de la santé publique du Québec. No individual-level socioeconomic variables are included in the RAMQ databases.

\section{Resource Utilization Bands (RUB)}

The RUB is calculated using The Johns Hopkins ACG System software. Patients are classified into 6 categories based on their health care services use, sex, age and diagnoses. Patients within each category are relatively homogeneous in terms of their predicted health care utilization in the following year:

- $\quad 0$-Non-users of services

- $\quad$ 1-Healthy users of services

- 2-Low Morbidity

- 3- Moderate Morbidity

- 4-High Morbidity

- 5-Very High Morbidity

The software also allows detecting patients with inconsistent data. They correspond to improbable cases, for example when clinical procedures intended only for women are attributed to men.

\section{Health status measured by the presence of different chronic conditions}

Diabetes, COPD, HF, hypertension, ischemic heart disease, arthritis, anxiety disorders and depression, schizophrenia, asthma

\section{Number of days of medication}

Total duration (in days) of drug prescriptions filled in a year.

\section{Usual provider of care index (UPC)}

The UPC index is calculated based on visits to general practitioners in private practice over2 years among patients with 3 or more visits. It indicates patients who have had $75 \%$ or more of their visits to the same physician over the past two years.

The variable UPC includes 3 categories:

- $\quad$ No usual provider of care - (UPC $<0.75)$

- Has a usual provider of care - (UPC $>=0.75)$

- $\quad$ Low users - (two or fewer visits during last two years)

Does enrollment in mltidisciplinary team-based primary care practice improve adherence to guidelinerecommended processes of care? Quebec's Family Medicine Groups, 2002-2010 M Diop, J Fiset-Laniel, S Provost, P Tousignant, RB Da Silva, MJ Ouimet. Health Policy 121 (4), 378-388 
Appendix 2: Exclusion criteria and patient characteristics at baseline (year prior to enrollment with GP)

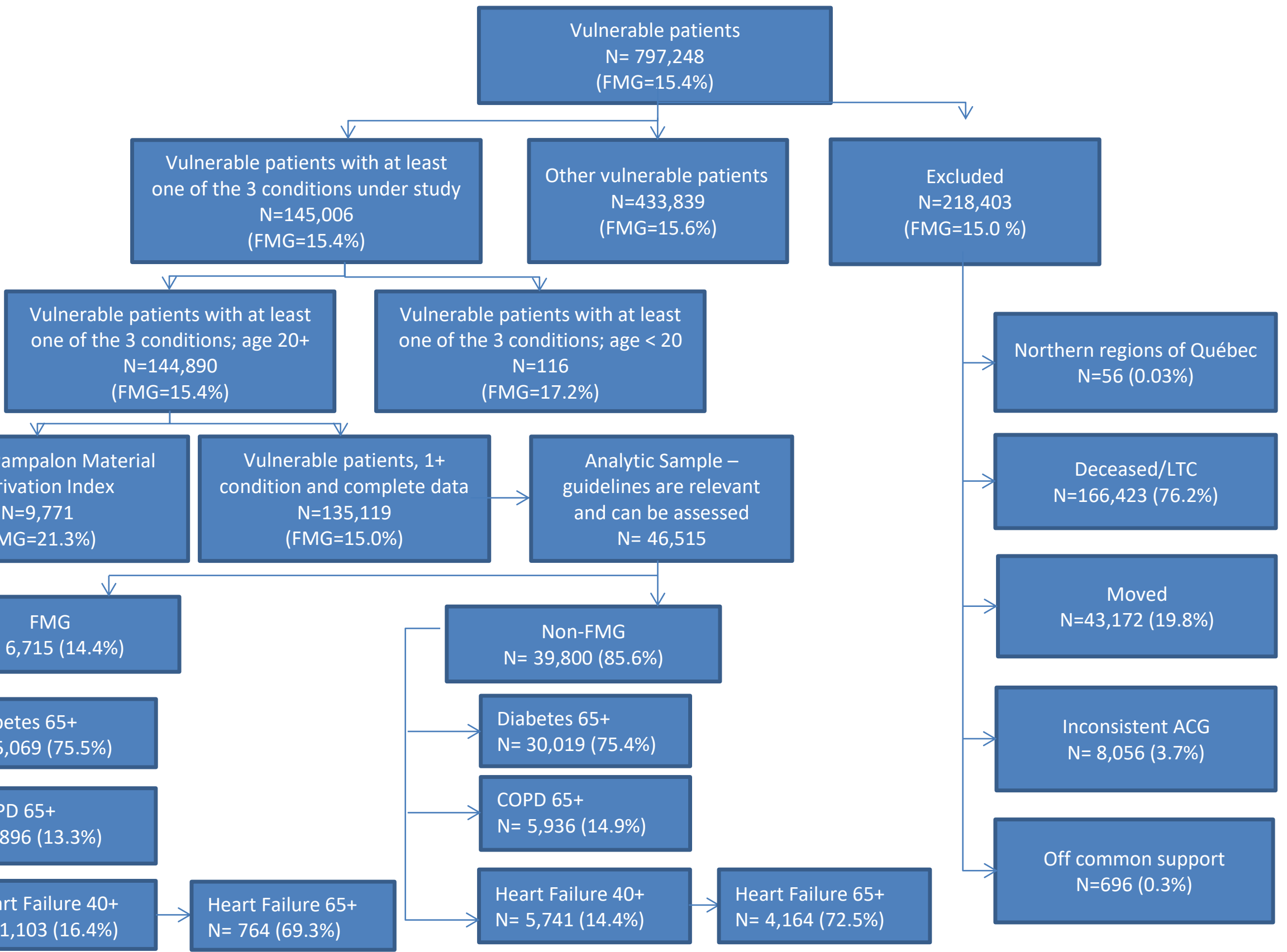

Does enrollment in mltidisciplinary team-based primary care practice improve adherence to guideline-recommended processes of care? Quebec's Family Medicine Groups, 2002-2010 M Diop, J Fiset-Laniel, S Provost, P Tousignant, RB Da Silva, MJ Ouimet. Health Policy 121 (4), 378-388 
Appendix 3: Algorithms to identify patients with chronic conditions

\begin{tabular}{ll}
\hline Disease & Algorithm \\
Diabetes & Within a two-year period, a patient \\
& with: \\
& 1) At least one diabetes diagnosis \\
& (primary or secondary) in hospital; OR \\
& 2) At least two diabetes diagnoses in \\
& physician billings.
\end{tabular}

Age Diagnostic Codes

ICD-9ii ICD-10

20 and 250

over

\section{E10 to E14}

Exclusions

To exclude cases of gestational diabetes, in-hospital diagnoses within 120 days before or 180 days after an obstetrical event are not considered.

These events begin with 641-676, V27 (ICD-9) or O10 to 019, 021 to 095, 098, 099, Z37 (ICD-10) for women between 10 and 54 years old inclusive. with:

1) At least one heart failure diagnosis (primary or secondary) in hospital; OR

2) At least two heart failure diagnoses in physician billings.

COPD

40 and $\quad 428 \quad 150$

over

50

35 and $491,492 \quad J 41$ to J44

Within a one-year period, a patient with:

1) At least one COPD diagnosis (primary

or secondary) in hospital; OR

2) At least one COPD diagnosis in

physician billings.
Identification Date

Cases identified based on date of hospital discharge or date of second physician billing.
Cases identified based on date of hospital discharge or date of second physician billing.

Cases identified based on date of hospital discharge or date of second physician billing.

\footnotetext{
i All diagnoses made in laboratories are excluded because they are not final diagnoses

ii International Classification of Diseases, 9th Revision

iii International Classification of Diseases, 10th Revision
} 
Appendix 4: Definition of guideline adherence indicators

\section{Disease-specific Indicators}

Diabetes

At least one ACEI or ARB prescription over two years

At least one statin prescription over two years

At least one visit with an optometrist or

ophthalmologist over two years

\section{Heart Failure}

At least one ACEI combination with a beta- blocker or ARB prescription over two years

At least one visit with a cardiologist or general practitioner over two years

COPD

At least one bronchodilator prescription over two years, among patients with prescription steroid

\section{Composite Indicators}

Prescriptions (4 possible guidelines)

- Adherence to $100 \%$ of guidelines

- Adherence to $75 \%$ of guidelines

- Adherence to $50 \%$ of guidelines

- Adherence to at least one guideline

Consultations ( 2 possible guidelines)

- Adherence to $100 \%$ of guidelines

- Adherence to at least one guideline

Prescriptions and consultations (6 possible guidelines)

- Adherence to $100 \%$ of guidelines

- Adherence to $75 \%$ of guidelines

- Adherence to $50 \%$ of guidelines

- Adherence to at least one guideline

\section{Population Definition}

Patients with diabetes at the time of enrollment with their GP, age 65 and older.

Patients with diabetes at the time of enrollment with their GP, age 65 and older.

Patients with diabetes at the time of enrollment with their GP, age 65 and older.

Patients with heart failure at the time of enrollment with their GP, age 65 and older.

Patients with heart failure at the time of enrollment with their GP, age 40 and older.

Patients with COPD and a steroid prescription at the time of enrollment with their GP, age 65 and older.

Patients with diabetes, COPD (with a steroid prescription), or heart failure at the time of enrollment with their GP, age 65 and older.

Patients with diabetes or heart failure at the time of enrollment with their GP, age 65 and 40 and older, respectively.

Patients with at diabetes, COPD (with a steroid prescription), or heart failure at the time of enrollment with their GP, age 65 and 40 and older, respectively.

Does enrollment in mltidisciplinary team-based primary care practice improve adherence to guidelinerecommended processes of care? Quebec's Family Medicine Groups, 2002-2010 M Diop, J Fiset-Laniel, S Provost, P Tousignant, RB Da Silva, MJ Ouimet. Health Policy 121 (4), 378-388 
Appendix 5: Baseline characteristics of patients in analysis sample

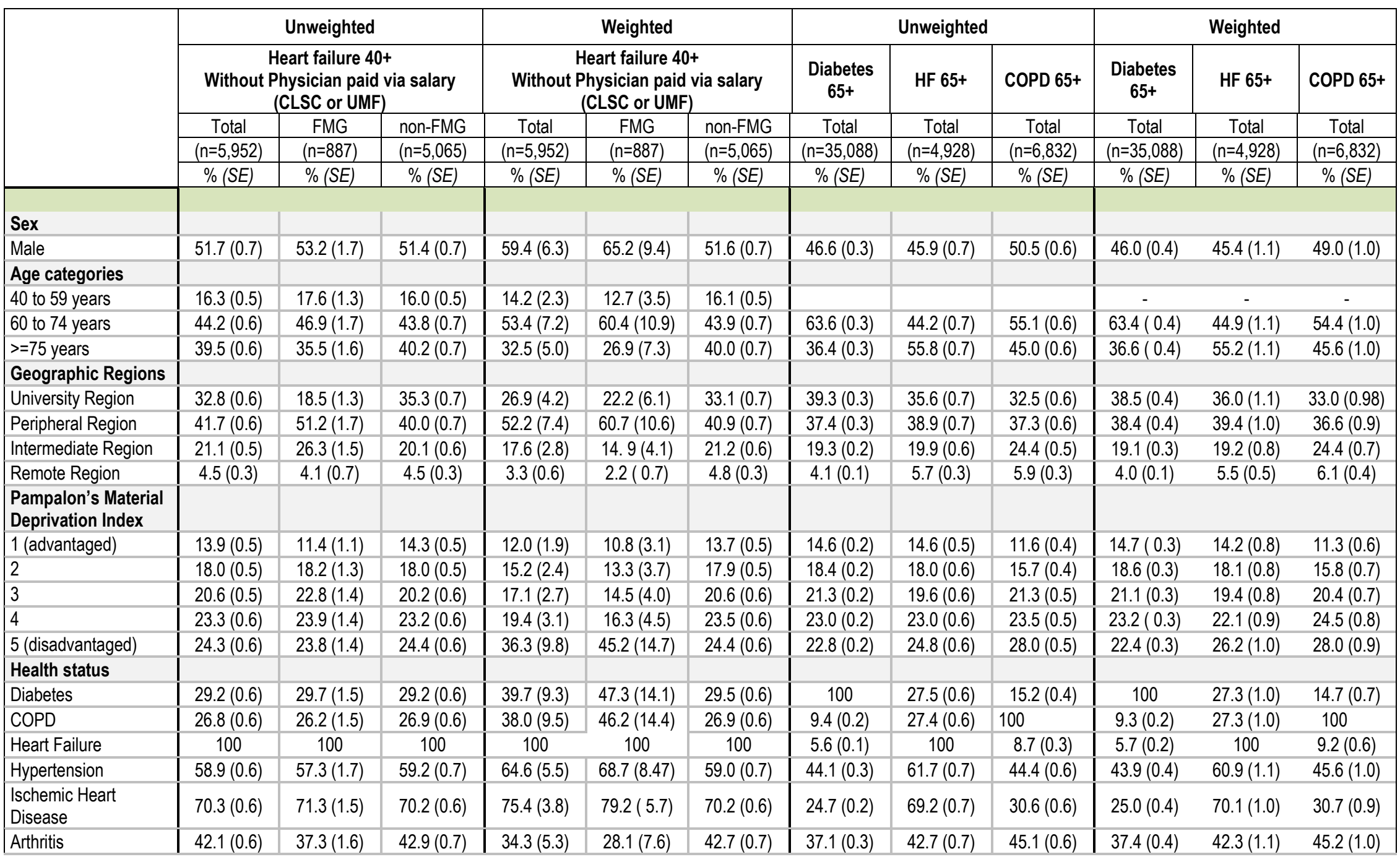

Does enrollment in mltidisciplinary team-based primary care practice improve adherence to guideline-recommended processes of care? Quebec's Family Medicine Groups, 2002-2010 M Diop, J Fiset-Laniel, S Provost, P Tousignant, RB Da Silva, MJ Ouimet. Health Policy 121 (4), $378-388$ 


\begin{tabular}{|c|c|c|c|c|c|c|c|c|c|c|c|c|}
\hline & \multirow{2}{*}{\multicolumn{3}{|c|}{$\begin{array}{c}\text { Unweighted } \\
\text { Heart failure } 40+ \\
\text { Without Physician paid via salary } \\
\text { (CLSC or UMF) }\end{array}$}} & \multirow{2}{*}{\multicolumn{3}{|c|}{$\begin{array}{c}\text { Weighted } \\
\text { Heart failure } 40+ \\
\text { Without Physician paid via salary } \\
\text { (CLSC or UMF) }\end{array}$}} & \multicolumn{3}{|c|}{ Unweighted } & \multicolumn{3}{|c|}{ Weighted } \\
\hline & & & & & & & \multirow{2}{*}{$\begin{array}{c}\begin{array}{c}\text { Diabetes } \\
65+\end{array} \\
\text { Total }\end{array}$} & \multirow{2}{*}{$\begin{array}{c}\text { HF 65+ } \\
\text { Total }\end{array}$} & \multirow{2}{*}{$\begin{array}{c}\text { COPD 65+ } \\
\text { Total }\end{array}$} & \multirow{2}{*}{$\begin{array}{c}\begin{array}{c}\text { Diabetes } \\
65+\end{array} \\
\text { Total }\end{array}$} & \multirow{2}{*}{$\begin{array}{c}\text { HF 65+ } \\
\text { Total }\end{array}$} & \multirow{2}{*}{$\begin{array}{c}\text { COPD 65+ } \\
\text { Total }\end{array}$} \\
\hline & Total & FMG & non-FMG & Total & FMG & non-FMG & & & & & & \\
\hline & $(n=5,952)$ & $(n=887)$ & $(n=5,065)$ & $(n=5,952)$ & $(n=887)$ & $(n=5,065)$ & $(n=35,088)$ & $(n=4,928)$ & $(n=6,832)$ & $(n=35,088)$ & $(n=4,928)$ & $(n=6,832)$ \\
\hline & $\%(S E)$ & $\%(S E)$ & $\%(S E)$ & $\%(S E)$ & $\%(S E)$ & $\%(S E)$ & $\%(S E)$ & $\%(S E)$ & $\%(S E)$ & $\%(S E)$ & $\%(S E)$ & $\%(S E)$ \\
\hline $\begin{array}{l}\text { Anxiety disorders and } \\
\text { depression }\end{array}$ & $12.1(0.4)$ & $10.3(1.0)$ & $12.4(0.5)$ & $9.7(1.6)$ & $7.9(2.3)$ & $12.3(0.5)$ & $7.9(0.1)$ & $10.7(0.4)$ & $12.9(0.4)$ & $7.5(0.2)$ & $10.6(0.7)$ & $12.5(0.6)$ \\
\hline Schizophrenia & $0.4(0.1)$ & $1.2(0.4)$ & $0.3(0.1)$ & $0.6(0.2)$ & $0.9(0.4)$ & $0.3(0.1)$ & $0.3(0.0)$ & $0.3(0.1)$ & $0.2(0.1)$ & $0.3(0.0)$ & $0.5(0.2)$ & $0.2(0.1)$ \\
\hline Asthma & $8.6(0.4)$ & $7.9(0.9)$ & $8.8(0.4)$ & $7.1(1.2)$ & $5.9(1.7)$ & $8.7(0.4)$ & $3.4(0.1)$ & $7.9(0.4)$ & $28.6(0.6)$ & $3.5(0.2)$ & $7.4(0.6)$ & $26.9(0.8)$ \\
\hline \multicolumn{13}{|c|}{ Resource Utilization Band (RUB) (mean) } \\
\hline $\begin{array}{l}\text { No use of health care } \\
\text { services }\end{array}$ & $0.7(0.1)$ & $0.7(0.3)$ & $0.7(0.1)$ & $0.6(0.2)$ & $0.5(0.3)$ & $0.8(0.1)$ & $0.9(0.1)$ & $1.0(0.1)$ & $0.9(0.1)$ & $0.9(0.1)$ & $0.9(0.2)$ & $1.1(0.2)$ \\
\hline $\begin{array}{l}\text { Healthy user of } \\
\text { services }\end{array}$ & $0.4(0.1)$ & $0.2(0.2)$ & $0.5(0.1)$ & $0.3(0.1)$ & $0.2(0.1)$ & $0.5(0.1)$ & $0.6(0.0)$ & $0.5(0.1)$ & $1.0(0.1)$ & $0.6(0.1)$ & $0.5(0.1)$ & $1.0(0.2)$ \\
\hline Light morbidity & $3.8(0.3)$ & $3.7(0.6)$ & $3.8(0.3)$ & $3.2(0.6)$ & $2.7(0.9)$ & $3.8(0.3)$ & $15.8(0.2)$ & $3.6(0.3)$ & $5.2(0.3)$ & $16.3(0.3)$ & $3.5(0.4)$ & $5.8(0.5)$ \\
\hline Moderate morbidity & $54.5(0.7)$ & $57.3(1.7)$ & $54.0(0.7)$ & $47.4(7.3)$ & $42.4(11.4)$ & $54.0(0.7)$ & $60.6(0.3)$ & $54.2(0.7)$ & $58.3(0.6)$ & $59.9(0.4)$ & $55.6(1.1)$ & $57.9(1.0)$ \\
\hline High morbidity & $24.4(0.6)$ & $24.1(1.4)$ & $24.4(0.6)$ & $20.6(3.2)$ & $17.9(4.9)$ & $24.3(0.6)$ & $14.1(0.2)$ & $24.0(0.6)$ & $22.0(0.5)$ & $14.5(0.3)$ & $23.8(0.9)$ & $22.1(0.8)$ \\
\hline Very high morbidity & $16.2(0.5)$ & $14.0(1.2)$ & $16.6(0.5)$ & $28.0(11.0)$ & $36.5(17.0)$ & $16.7(0.5)$ & $8.0(0.2)$ & $16.6(0.5)$ & $12.5(0.4)$ & $7.7(0.2)$ & $15.8(0.8)$ & $12.2(0.6)$ \\
\hline \multicolumn{13}{|c|}{ Tertiary Health Service Utilization (mean) } \\
\hline $\begin{array}{l}\text { Number of Emergency } \\
\text { Room visits }\end{array}$ & $1.2(0.0)$ & $1.2(0.1)$ & $1.2(0.0)$ & $1.8(0.5)$ & $2.2(0.8)$ & $1.2(0.0)$ & $0.6(0.0)$ & $1.1(0.0)$ & $1.2(0.0)$ & $0.6(0.0)$ & $1.2(0.1)$ & $1.2(0.0)$ \\
\hline $\begin{array}{l}\text { Number of ER visits } \\
\text { for ambulatory care } \\
\text { sensitive conditions }\end{array}$ & $0.0(0.0)$ & $0.0(0.0)$ & $0.0(0.0)$ & $0.0(0.0)$ & $0.0(0.0)$ & $0.0(0.0)$ & $0.0(0.0)$ & $0.0(0.0)$ & $0.0(0.0)$ & $0.0(0.0)$ & $0.0(0.0)$ & $0.0(0.0)$ \\
\hline $\begin{array}{l}\text { Number of ER visits } \\
\text { with hospitalization }\end{array}$ & $0.2(0.0)$ & $0.2(0.0)$ & $0.2(0.0)$ & $0.2(0.0)$ & $0.1(0.0)$ & $0.2(0.0)$ & $0.1(0.0)$ & $0.2(0.0)$ & $0.2(0.0)$ & $0.1(0.0)$ & $0.2(0.0)$ & $0.2(0.0)$ \\
\hline $\begin{array}{l}\text { Number of } \\
\text { hospitalizations }\end{array}$ & $0.4(0.0)$ & $0.4(0.0)$ & $0.4(0.0)$ & $0.5(0.1)$ & $0.5(0.1)$ & $0.4(0.0)$ & $0.2(0.0)$ & $0.4(0.0)$ & $0.4(0.0)$ & $20.2(0.5)$ & $0.4(0.0)$ & $0.4(0.0)$ \\
\hline $\begin{array}{l}\text { Number of } \\
\text { hospitalizations for } \\
\text { ambulatory care } \\
\text { sensitive conditions }\end{array}$ & $0.1(0.0)$ & $0.1(0.0)$ & $0.1(0.0)$ & $0.1(0.0)$ & $0.1(0.0)$ & $0.1(0.0)$ & $0.0(0.0)$ & $0.1(0.0)$ & $0.1(0.0)$ & $2.3(0.2)$ & $0.1(0.0)$ & $0.1(0.0)$ \\
\hline \multicolumn{13}{|c|}{ Ambulatory Health Service Utilization (mean) } \\
\hline $\begin{array}{l}\text { Number of ambulatory } \\
\text { consultations (All) }\end{array}$ & $14.6(0.2)$ & $12.8(0.4)$ & $14.9(0.2)$ & $47.6(28.4)$ & $72.1(43.0)$ & $14.7(0.2)$ & $10.90 .0)$ & $14.1(0.2)$ & $13.2(0.1)$ & $10.9(0.1)$ & $14.2(0.3)$ & $13.1(0.2)$ \\
\hline
\end{tabular}

Does enrollment in mltidisciplinary team-based primary care practice improve adherence to guideline-recommended processes of care? Quebec's Family Medicine Groups, 2002-2010 M Diop, J Fiset-Laniel, S Provost, P Tousignant, RB Da Silva, MJ Ouimet. Health Policy 121 (4), $378-388$ 


\begin{tabular}{|c|c|c|c|c|c|c|c|c|c|c|c|c|}
\hline & \multirow{2}{*}{\multicolumn{3}{|c|}{$\begin{array}{c}\text { Unweighted } \\
\text { Heart failure } 40+ \\
\text { Without Physician paid via salary } \\
\text { (CLSC or UMF) }\end{array}$}} & \multirow{2}{*}{\multicolumn{3}{|c|}{$\begin{array}{c}\text { Weighted } \\
\text { Heart failure } 40+ \\
\text { Without Physician paid via salary } \\
\text { (CLSC or UMF) }\end{array}$}} & \multicolumn{3}{|c|}{ Unweighted } & \multicolumn{3}{|c|}{ Weighted } \\
\hline & & & & & & & \multirow{2}{*}{$\begin{array}{c}\begin{array}{c}\text { Diabetes } \\
65+\end{array} \\
\text { Total }\end{array}$} & \multirow{2}{*}{$\begin{array}{c}\text { HF 65+ } \\
\text { Total }\end{array}$} & \multirow{2}{*}{$\begin{array}{c}\text { COPD 65+ } \\
\text { Total }\end{array}$} & \multirow{2}{*}{$\begin{array}{c}\begin{array}{c}\text { Diabetes } \\
65+\end{array} \\
\text { Total }\end{array}$} & \multirow{2}{*}{$\begin{array}{c}\text { HF 65+ } \\
\text { Total } \\
\end{array}$} & \multirow{2}{*}{$\begin{array}{c}\text { COPD 65+ } \\
\text { Total }\end{array}$} \\
\hline & Total & FMG & non-FMG & Total & FMG & non-FMG & & & & & & \\
\hline & $(n=5,952)$ & $(n=887)$ & $(n=5,065)$ & $(n=5,952)$ & $(n=887)$ & $(n=5,065)$ & $(n=35,088)$ & $(n=4,928)$ & $(n=6,832)$ & $(n=35,088)$ & $(n=4,928)$ & $(n=6,832)$ \\
\hline & $\%(S E)$ & $\%(S E)$ & $\%(S E)$ & $\%(S E)$ & $\%(S E)$ & $\%(S E)$ & $\%(S E)$ & $\%$ (SE) & $\%(S E)$ & $\%(S E)$ & $\%(S E)$ & $\%(S E)$ \\
\hline $\begin{array}{l}\text { Number of } \\
\text { consultations } \\
\text { (Generalist) }\end{array}$ & $7.5(0.1)$ & $6.8(0.2)$ & $7.6(0.1)$ & $8.6(1.0)$ & $9.4(1.5)$ & $7.5(0.1)$ & $5.8(0.0)$ & $7.3(0.1)$ & $7.5(0.1)$ & $5.8(0.0)$ & $7.3(0.3)$ & $7.5(0.1)$ \\
\hline $\begin{array}{l}\text { Number of } \\
\text { consultations } \\
\text { (Specialist) }\end{array}$ & $7.1(0.1)$ & $6.0(0.3)$ & $7.3(0.1)$ & $39.0(27.4)$ & $62.8(41.5)$ & $7.2(0.1)$ & $5.1(0.0)$ & $6.8(0.1)$ & $5.7(0.1)$ & $5.1(0.1)$ & $6.9(0.2)$ & $5.6(0.1)$ \\
\hline $\begin{array}{l}\text { Number of different } \\
\text { physicians seen }\end{array}$ & $6.5(0.1)$ & $6.0(0.1)$ & $6.6(0.1)$ & $8.8(2.0)$ & 10.5 (3.1) & $6.6(0.1)$ & $5.0(0.0)$ & $6.4(0.1)$ & $6.1(0.1)$ & $5.0(0.0)$ & $6.4(0.1)$ & $6.1(0.1)$ \\
\hline $\begin{array}{l}\text { Number of different } \\
\text { generalists seen }\end{array}$ & $2.7(0.0)$ & $2.6(0.1)$ & $2.7(0.0)$ & $3.5(0.7)$ & $4.1(1.1)$ & $2.7(0.0)$ & $2.1(0.0)$ & $2.6(0.0)$ & $2.9(0.0)$ & $2.1(0.0)$ & $2.6(0.1)$ & $2.9(0.0)$ \\
\hline $\begin{array}{l}\text { Number of different } \\
\text { specialists seen }\end{array}$ & $3.9(0.0)$ & $3.4(0.1)$ & $4.0(0.1)$ & $5.3(1.3)$ & $6.4(2.0)$ & $3.9(0.1)$ & $2.9(0.0)$ & $3.8(0.0)$ & $3.2(0.0)$ & $2.9(0.0)$ & $3.8(0.1)$ & $3.2(0.1)$ \\
\hline \multicolumn{13}{|c|}{ Usual providers of care (UPC) } \\
\hline $\begin{array}{l}\text { No usual provider of } \\
\text { care - }(\text { UPC }<0.75)\end{array}$ & $18.6(0.5)$ & $21.4(1.4)$ & $18.1(0.5)$ & $16.2(2.6)$ & $14.50(4.0)$ & $18.5(0.6)$ & $17.5(0.2)$ & $17.4(0.5)$ & $24.4(0.5)$ & $16.7(0.3)$ & $17.7(0.8)$ & $24.3(0.8)$ \\
\hline $\begin{array}{l}\text { With usual provider of } \\
\text { care }-(\text { UPC }>=0.75)\end{array}$ & $76.9(0.6)$ & $72.6(1.5)$ & $77.7(0.6)$ & $80.3(3.1)$ & $82.8(4.7)$ & $76.9(0.6)$ & $77.4(0.2)$ & $73.8(0.6)$ & $68.8(0.6)$ & $78.3(0.3)$ & $73.2(0.9)$ & $68.6(0.9)$ \\
\hline $\begin{array}{l}\text { Low users - (two or } \\
\text { less visits during last } \\
\text { two years) }\end{array}$ & $4.5(0.3)$ & $6.0(0.8)$ & $4.2(0.3)$ & $3.5(0.6)$ & $2.8(0.8)$ & $4.6(0.3)$ & $5.1(0.1)$ & $8.8(0.4)$ & $6.9(0.3)$ & $5.1(0.2)$ & $9.1(0.5)$ & $7.2(0.4)$ \\
\hline \multicolumn{13}{|c|}{ Additional coverage RAMQ } \\
\hline yes & $95.9(0.3)$ & $96.2(0.8)$ & $95.9(0.3)$ & $95.3(0.6)$ & $94.5(1.2)$ & $96.1(0.3)$ & $95.0(0.1)$ & $96.5(0.3)$ & 100 & $94.5(0.2)$ & $96.1(0.5)$ & 100 \\
\hline \multicolumn{13}{|c|}{ Physician paid via salary (CLSC or UMF) } \\
\hline Yes & - & - & - & - & - & - & $10.6 \quad 0.2$ & $13.7 \quad 0.5$ & $11.7 \quad 0.4$ & $12.7(0.3)$ & $16.0(0.8)$ & $13.5(0.7)$ \\
\hline \multicolumn{13}{|c|}{ Care consistent with clinical guidelines } \\
\hline $\begin{array}{l}\text { Diabetes-At least one } \\
\text { prescription IECA or } \\
\text { ARA over two years }\end{array}$ & & & & & & & $69.7(0.2)$ & & & $69.7(0.4)$ & & \\
\hline $\begin{array}{l}\text { Diabetes-At least one } \\
\text { prescription Statin } \\
\text { over two years }\end{array}$ & & & & & & & $54.2(0.3)$ & & & $54.5(0.4)$ & & \\
\hline
\end{tabular}

Does enrollment in mltidisciplinary team-based primary care practice improve adherence to guideline-recommended processes of care? Quebec's Family Medicine Groups, 2002-2010 M Diop, J Fiset-Laniel, S Provost, P Tousignant, RB Da Silva, MJ Ouimet. Health Policy 121 (4), $378-388$ 


\begin{tabular}{|c|c|c|c|c|c|c|c|c|c|c|c|c|}
\hline & \multirow{2}{*}{\multicolumn{3}{|c|}{$\begin{array}{c}\text { Unweighted } \\
\text { Heart failure } 40+ \\
\text { Without Physician paid via salary } \\
\text { (CLSC or UMF) }\end{array}$}} & \multirow{2}{*}{\multicolumn{3}{|c|}{$\begin{array}{c}\text { Weighted } \\
\text { Heart failure } 40+ \\
\text { Without Physician paid via salary } \\
\text { (CLSC or UMF) }\end{array}$}} & \multicolumn{3}{|c|}{ Unweighted } & \multicolumn{3}{|c|}{ Weighted } \\
\hline & & & & & & & \multirow{2}{*}{$\begin{array}{c}\begin{array}{c}\text { Diabetes } \\
65+\end{array} \\
\text { Total }\end{array}$} & \multirow{2}{*}{$\begin{array}{c}\text { HF 65+ } \\
\text { Total }\end{array}$} & \multirow{2}{*}{$\begin{array}{c}\text { COPD 65+ } \\
\text { Total }\end{array}$} & \multirow{2}{*}{$\begin{array}{c}\begin{array}{c}\text { Diabetes } \\
65+\end{array} \\
\text { Total }\end{array}$} & \multirow{2}{*}{$\begin{array}{c}\text { HF 65+ } \\
\text { Total }\end{array}$} & \multirow{2}{*}{$\begin{array}{c}\text { COPD 65+ } \\
\text { Total }\end{array}$} \\
\hline & Total & FMG & non-FMG & Total & FMG & non-FMG & & & & & & \\
\hline & $(n=5,952)$ & $(n=887)$ & $(n=5,065)$ & $(n=5,952)$ & $(n=887)$ & $(n=5,065)$ & $(n=35,088)$ & $(n=4,928)$ & $(n=6,832)$ & $(n=35,088)$ & $(n=4,928)$ & $(n=6,832)$ \\
\hline & $\%(S E)$ & $\%(S E)$ & $\%(S E)$ & $\%(S E)$ & $\%(S E)$ & $\%(S E)$ & $\%(S E)$ & $\%(S E)$ & $\%(S E)$ & $\%(S E)$ & $\%(S E)$ & $\%(S E)$ \\
\hline $\begin{array}{l}\text { Diabetes- At least one } \\
\text { ophthalmologist or } \\
\text { optometrist } \\
\text { consultation over two } \\
\text { years }\end{array}$ & & & & & & & $84.4(0.2)$ & & & $84.2(0.3)$ & & \\
\hline $\begin{array}{l}\text { Heart failure -At least } \\
\text { one prescription IECA } \\
\text { or ARA over two years }\end{array}$ & $61.7(0.7)$ & $63.7(2.0)$ & $61.4(0.8)$ & $60.7(1.2)$ & $59.4(2.2)$ & $61.9(0.8)$ & & $62.1(0.7)$ & & & $61.6(1.1)$ & \\
\hline $\begin{array}{l}\text { Heart failure -At least } \\
\text { one GP or cardiologist } \\
\text { consultation over two } \\
\text { years }\end{array}$ & $99.8(0.1)$ & $99.8(0.2)$ & $99.9(0.1)$ & $99.9(0.0)$ & $99.9(0.1)$ & $99.9(0.1)$ & & $98.8(0.2)$ & & & $98.6(0.2)$ & \\
\hline $\begin{array}{l}\text { COPD-Patients with } \\
\text { prescription steroid- At } \\
\text { least one prescription } \\
\text { bronchodilator over } \\
\text { two year }\end{array}$ & & & & & & & & & $96.2(0.2)$ & & & $96.4(0.4)$ \\
\hline
\end{tabular}

Does enrollment in mltidisciplinary team-based primary care practice improve adherence to guideline-recommended processes of care? Quebec's Family Medicine Groups, 2002-2010 M Diop, J Fiset-Laniel, S Provost, P Tousignant, RB Da Silva, MJ Ouimet. Health Policy 121 (4), $378-388$ 